\title{
Carbon catabolite repression involves physical interaction of the transcription factor CRE1/CreA and the Tup1-Cyc8 complex in Penicillium oxalicum and Trichoderma reesei
}

Yueyan Hu${ }^{1,2,3}$, Mengxue Li ${ }^{1}$, Zhongjiao Liu' ${ }^{1}$, Xin Song ${ }^{1,2}$, Yinbo Qu ${ }^{1,2}$ and Yuqi Qin ${ }^{1,2,3^{*}}$ (D)

\begin{abstract}
Background: Cellulolytic enzyme production in filamentous fungi requires a release from carbon catabolite repression (CCR). The protein CRE1/CreA (CRE= catabolite responsive element) is a key transcription factor (TF) that is involved in CCR and represses cellulolytic gene expression. CRE1/CreA represents the functional equivalent of Mig $1 \mathrm{p}$, an important Saccharomyces cerevisiae TF in CCR that exerts its repressive effect by recruiting a corepressor complex Tup 1p-Cyc8p. Although it is known from S. cerevisiae that CRE1/CreA might repress gene expression via interacting with the corepressor complex Tup1-Cyc8, this mechanism is unconfirmed in other filamentous fungi, since the physical interaction has not yet been verified in these organisms. The precise mechanism on how CRE1/CreA achieves transcriptional repression after DNA binding remains unknown.

Results: The results from tandem affinity purification and bimolecular fluorescence complementation revealed a direct physical interaction between the TF CRE1/CreA and the complex Tup1-Cyc8 in the nucleus of cellulolytic fungus Trichoderma reesei and Penicillium oxalicum. Both fungi have the ability to secrete a complex arsenal of enzymes to synergistically degrade lignocellulosic materials. In P. oxalicum, the protein PoCyc8, a subunit of complex Tup1Cyc8, interacts directly with TF PoCreA and histone H3 lysine 36 (H3K36) methyltransferase PoSet2 in the nucleus. The di-methylation level of H3K36 in the promoter of prominent cellulolytic genes (cellobiohydrolase-encoding gene Pocbh1/cel7A and endoglucanase-encoding gene Poeg/1/cel7B) is positively correlated with the expression levels of TF PoCreA. Since the methylation of H3K36 was also demonstrated to be a repression marker of cellulolytic gene expression, it appears feasible that the cellulolytic genes are repressed via PoCreA-Tup1-Cyc8-Set2-mediated transcriptional repression.
\end{abstract}

Conclusion: This study verifies the long-standing conjecture that the TF CRE1/CreA represses gene expression by interacting with the corepressor complex Tup1-Cyc8 in filamentous fungi. A reasonable explanation is proposed that PoCreA represses gene expression by recruiting complex PoTup1-Cyc8. Histone methyltransferase Set2, which methylates $\mathrm{H3K} 36$, is also involved in the regulatory network by interacting with PoCyc8. The findings contribute to the understanding of CCR mechanism in filamentous fungi and could aid in biotechnologically relevant enzyme production.

\footnotetext{
*Correspondence: qinyuqi@sdu.edu.cn

${ }^{1}$ National Glycoengineering Research Center, State Key Laboratory

of Microbial Technology, Shandong University, No. 72 Binhai Road, Qingdao 266237, China

Full list of author information is available at the end of the article
} original author(s) and the source, provide a link to the Creative Commons licence, and indicate if changes were made. The images or other third party material in this article are included in the article's Creative Commons licence, unless indicated otherwise in a credit line to the material. If material is not included in the article's Creative Commons licence and your intended use is not permitted by statutory regulation or exceeds the permitted use, you will need to obtain permission directly from the copyright holder. To view a copy of this licence, visit http://creativecommons.org/licenses/by/4.0/. The Creative Commons Public Domain Dedication waiver (http://creativeco mmons.org/publicdomain/zero/1.0/) applies to the data made available in this article, unless otherwise stated in a credit line to the data. 
Keywords: CCR, Cellulases, CRE1, Penicillium, Transcription factor, Trichoderma

\section{Introduction}

Lignocellulosic biomass composed of polysaccharides (cellulose and hemicellulose) and an aromatic polymer (lignin) is the most abundant and highly renewable natural biological resource [1]. Many saprophytic fungi secrete different types of cellulolytic enzymes that degrade cellulose and hemicelluloses to a mixture of sugars (C-5 and C-6), which are then assimilated and metabolized by different microorganisms to participate in the global carbon cycle [2]. These sugars can also be fermented by industrial microorganisms to produce various chemicals, such as alcohols and organic acids [3, 4].

Cellulolytic enzyme production in filamentous fungi is tightly controlled at the transcription level. Cellulolytic gene expression is often repressed in the presence of preferentially utilized sugars (frequently glucose), a phenomenon known as carbon catabolite repression (CCR) [5]. In cellulolytic fungi, e.g., Trichoderma, Aspergillus, Neurospora, and Penicillium, CCR is mediated mainly by the transcription factor (TF) $\mathrm{CRE} 1 / \mathrm{CreA}(\mathrm{CRE}=$ catabolite responsive element), a $\mathrm{C} 2 \mathrm{H} 2$ zinc finger protein that binds to the promoters of various genes repressed by glucose or xylose [6-8]. In Aspergillus nidulans and Trichoderma reesei, CRE1/CreA directly binds to 5'-SYGGRG-3' motif in the proximal promoter region and inhibits the expression of xylanase-encoding genes such as $x \ln A, x \ln B$, and $x \ln D$ [9] and cellulase-encoding genes such as cbh1 (cel7A), cbh2 (cel6A), and eg2 (cel5A) $[5,10,11]$. Alterations in the subcellular localization of CRE1/CreA mediated by glucose concentration and posttranslational modification (specifically phosphorylation) are crucial for its regulation [12-14].

Cellulolytic gene induction requires a release from CCR. Therefore, the deletion, truncation, or multisitedirected mutagenesis of gene $\mathrm{cre} 1 / \mathrm{cre} A$ can alleviate CCR and thus improve the expression level of prominent cellulolytic genes in various carbon sources, such as glucose, lactose, sophorose, cellulose, or a mixture of plant polymers [5, 15-18]. For example, either the deletion or truncation of cre1 in T. reesei wild-type strain QM6a leads to de-repressed production of cellulase and hemicellulase, when the mutants are cultivated in glucose-containing media [19]. The hypercellulolytic T. reesei strain RutC30, which can produce cellulase and hemicellulase in a medium containing glucose, has a truncated version of TrCRE1 [20]. Another hyperproducer of cellulolytic enzyme, Penicillium oxalicum JU-A10-T, has a frameshift mutation at the $\mathrm{C}$-terminus of PoCreA, which plays a negative role on cellulolytic gene expression under repressed (glucose) or induced (cellulose) condition [8]. In addition, CRE1/CreA is crucial in many other biological processes, including asexual development, secondary metabolite production, glycogen metabolism, fungal virulence, and circadian rhythms in diverse fungi [21-24].

The regulating function of CRE1/CreA for the above biological processes ultimately originates from its controlling (specifically repression) roles for gene expression. However, the precise mechanism of transcriptional repression by CRE1/CreA after DNA binding remains unknown. The amino acid sequence of CRE1/CreA zinc finger region is similar to that of budding yeast Mig1p, an important TF in CCR [25]. In Saccharomyces cerevisiae, Mig1p exerts its repressive effect by recruiting corepressor complex Tup1p-Cyc8p (Ssn6) [26]. In filamentous fungi, Tup1p and Cyc8p have conserved homologous proteins, such as RcoA and SsnF in A. nidulans, RCO-1 and RCM-1 in Neurospora crassa, and TrTUP1 and TrCYC8 in T. reesei [21, 27-30]. Although it is known from $S$. cerevisiae that CRE1/CreA might repress gene expression via interacting with the corepressor complex Tup1-Cyc8, this mechanism is unconfirmed in other filamentous fungi, since the physical interaction between CRE1/CreA and the complex has not yet been verified in these organisms. $N$. crassa CRE- $1, \mathrm{RCO}-1$, and RCM-1 proteins are involved in fungal development, glycogen accumulation, and phosphorylation-regulated glycogen synthase activity. However, whether $N$. crassa CRE-1 recruits the complex RCO-1/RCM-1 has not been proven $[21,29]$. T. reesei TrTUP1 or TrCYC8 knockdown does not result in carbon catabolite de-repression [30]. García et al. showed that the absence of $r c o A$ (the homologue of yeast Tup1p) does not cause carbon catabolite de-repression in $A$. nidulans [27]. The cohesive picture of gene repression mediated by CRE1/CreA in filamentous fungi has never been explored.

In this study, tandem affinity purification (TAP) and bimolecular fluorescence complementation (BiFC) were used to verify the direct physical interaction between the TF CRE1/CreA and the complex Tup1-Cyc8 in T. reesei and $P$. oxalicum. A reasonable explanation on how PoCreA represses gene expression by recruiting Tup1Cyc8 was also presented.

\section{Results}

TrTUP1 and TrCYC8 are protein-protein interaction partners of $T$. reesei TrCRE1

Eukaryotic TFs regulate transcription by recruiting cofactors that control the specific phases of transcription. 
TAP is a purification technique for protein-protein interaction analysis that incorporates an epitope tag (TAP tag) onto the protein of interest and performs a two-step affinity purification protocol to isolate TAPtagged proteins and associated proteins. This two-step purification process reduces the amount of non-specific binding proteins. TAP coupled with mass-spectrometry (TAP-MS) for CRE1/CreA was performed in T. reesei and $P$. oxalicum to identify the putative cofactors of CRE1/CreA. First, TAP-MS for T. reesei TrCRE1-labeled strain (TrCRE1-FLAG-HA) was conducted to identify the protein-protein interaction collaborator of TrCRE1. The gene encoding for TrCRE1 was C-terminally fused with the FLAG (DYKDDDDK) and HA (YPYDVPDYA) tags (theoretical molecular weight (MW): $4.28 \mathrm{kDa}$ ) and then transformed into the parent strain T. reesei QP4 [31] to substitute the native Trcre1 gene. The corresponding strain was named TrCRE1-TAP. The strains are listed in Additional file 1: Table S1.

No significant difference in mycelia growth and conidia production was observed between the TrCRE1-TAP and the parent strain. In particular, their cellulolytic genes had a similar expression pattern (Additional file 2: Fig. S1A, B), indicating the lack of biological interference from the insertion of FLAG and HA tags. For TAP-MS experiments, TAP eluents from the parent strain T. reesei QP4 were used as the control. The final TAP eluents from the respective strains were divided into three parts for Western blot analysis, SDS-PAGE with silver staining, and LC-MS/MS to identify the bait and interacting proteins.

Western blot analysis indicated the existence of TrCRE1 bait (Fig. 1A). Several specific bands were found between the TrCRE1-TAP and its parent strain QP4 from the gel of SDS-PAGE with subsequent silver staining (Fig. 1B). The bands were cut from the gel and identified by LC/MS-MS as TrCRE1 (Fig. 1B, red arrow, approximately $50 \mathrm{kDa}$, theoretical MW: $43.62 \mathrm{kDa}$ ), TrTUP1 (Fig. 1B, green arrow, approximately $70 \mathrm{kDa}$; theoretical MW: $66.00 \mathrm{kDa}$ ), and TrCYC8 (Fig. 1B, blue arrow, approximately $115 \mathrm{kDa}$, theoretical MW: $82.06 \mathrm{kDa}$ ). The proteins in the final eluent were identified by LC-MS/ MS, and the TAP eluents from the parent strain T. reese $i$ QP4 were used as the control. The proteins in all three TrCRE1-TAP samples but not in any of the controls were considered putative interacting proteins.

In addition to TrCRE1 itself as the bait, 37 protein targets of putative interactions with TrCRE1 were captured (Additional file 3: Spreadsheet S1). The top 10 proteins with the highest exponentially modified protein abundance index (emPAI) [32] are listed in Table 1. Among the top 10 proteins, TrCRE1 is the top one protein according to emPAI, TrTUP1 (Trire2_121940, the homologue of S. cerevisiae Tup1p) and TrCYC8 (Trire2_102616, the homologue of $S$. cerevisiae Cyc8p) are listed in the 3rd and 4th positions, respectively. Except for the complex, other putative proteins were listed in Table 1. These homologues of putative interacting proteins in S. cerevisiae are not completely localized in the nucleus. This finding is reasonable because CRE1/CreA is distributed in the nucleus and cytoplasm, even under repressed (glucose) condition [14, 33].

\section{PoTup1 and PoCyc8 are also protein-protein interaction partners of $P$. oxalicum PoCreA}

P. oxalicum PoCreA-TAP strain was constructed using the same method for $T$. reese $i$, and the strains are listed in Additional file 1: Table S1. No significant difference in mycelia growth and conidia production was observed between the PoCreA-TAP and the parent strain. In particular, their cellulolytic genes had a similar expression pattern (Additional file 2: Fig. S1C, D), indicating the lack of biological interference from the insertion of FLAG and HA tags. Western blot analysis indicated the existence of PoCreA bait (approximately $50 \mathrm{kDa}$; theoretical MW: $44.95 \mathrm{kDa}$ ) (Fig. 1C), although no specific band was observed between the samples of PoCreA-TAP and parent strain 114-2 from the gel of SDS-PAGE with subsequent silver staining (Fig. 1D). The proteins in the final eluent were identified by LC-MS/MS, and the TAP eluents from the parent strain $P$. oxalicum 114-2 were used as the control. The proteins in all three PoCreA-TAP samples but not in any of the controls were considered putative interacting proteins.

In addition to PoCreA itself, 21 protein targets of putative interactions with PoCreA were captured (Additional file 3: Spreadsheet S1). The top 10 proteins with the highest emPAI are listed in Table 2. Among the top 10 proteins, the bait PoCreA showed the highest emPAI followed by PoTup1 (PDE_01024, the homologue of S. cerevisiae Tup1p) and PoCyc8 (PDE_03177, the homologue of $S$. cerevisiae Cyc8p) (Table 2). The finding indicates that the PoTup1-Cyc8 complex is the main interactor for PoCreA. On the basis of previous silver staining results and TAP-MS experiment for PoCreA, PoTup1 and PoCyc8 are considered as the putative interacting proteins of PoCreA under glucose condition.

\section{PoCreA was observed in the protein-protein interaction of $P$. oxalicum PoCyc8}

PoCyc8-TAP strain was constructed using the same method for T. reesei. The strains are listed in Additional file 1: Table S1. No significant difference in mycelia growth and conidia production was observed between the PoCyc8-TAP and the parent strain. In particular, their cellulolytic gene had a similar expression pattern 


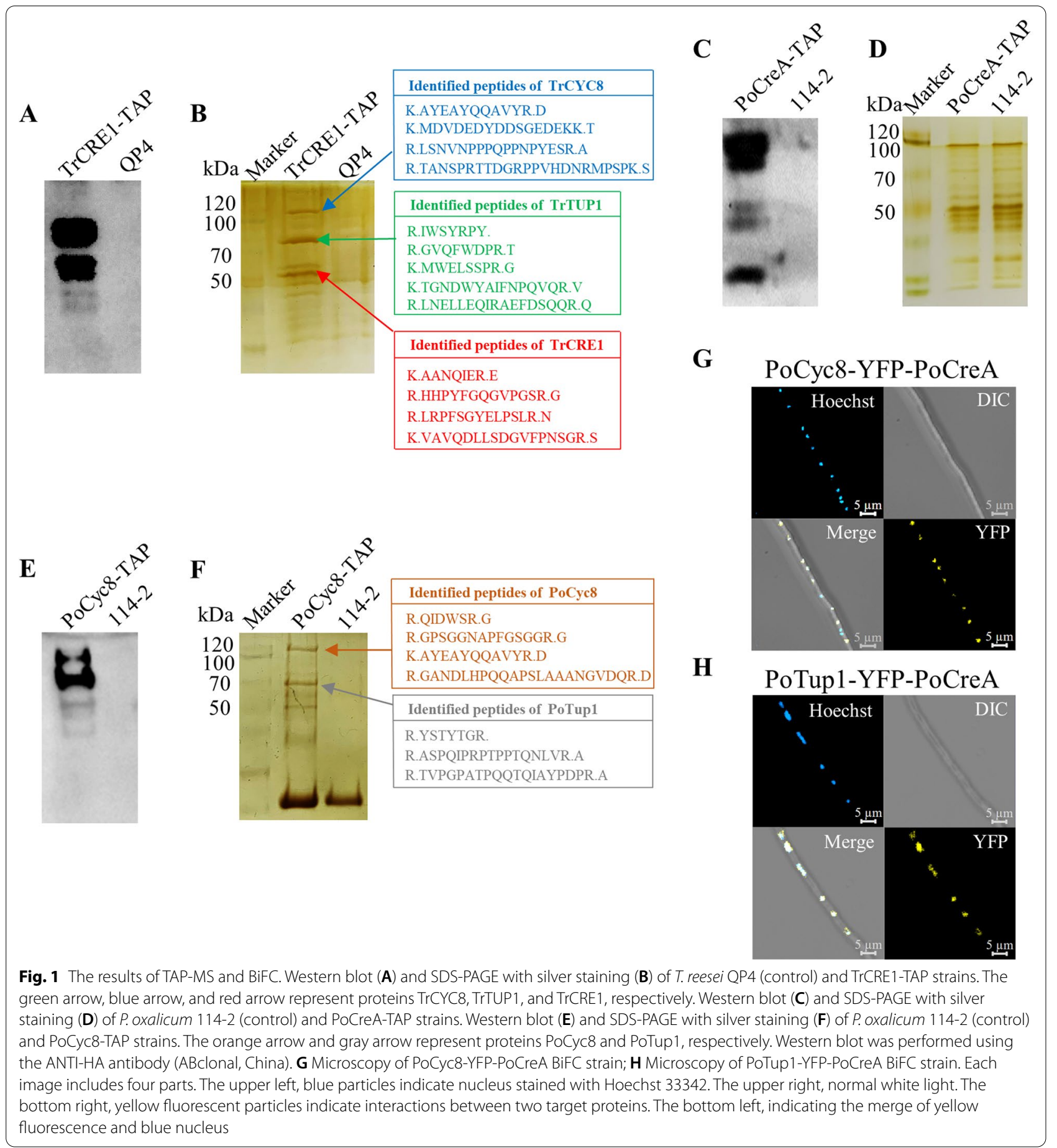

(Additional file 2: Fig. S1C, D). Western blot analysis indicated the existence of PoCyc8 bait (Fig. 1E). Several specific bands were found between the PoCyc8-TAP and its parent strain 114-2 from the gel of SDS-PAGE with subsequent silver staining (Fig. 1F). The bands were cut from the gel and identified by LC-MS/MS as PoCyc8 (Fig. 1F, orange arrow, approximately $115 \mathrm{kDa}$, theoretical MW:
95.27 kDa) and PoTup1 (Fig. 1F, gray arrow, approximately $70 \mathrm{kDa}$; theoretical MW: $63.81 \mathrm{kDa}$ ). The proteins in the final eluent were identified by LC-MS/MS, and the TAP eluents from the parent strain $P$. oxalicum 114-2 were used as the control. The proteins in all three PoCyc8-TAP samples but not in any of the controls were considered putative interacting proteins. 
Table 1 The top 10 proteins interacting with TrCRE1 identified through TAP-MS

\begin{tabular}{|c|c|c|c|c|c|c|c|c|}
\hline \multirow[t]{2}{*}{ Rank } & \multirow[t]{2}{*}{ Protein ID } & \multirow[t]{2}{*}{ emPAl $^{\mathrm{a}}$} & \multirow{2}{*}{$\begin{array}{l}\text { T. reesei QM6a } \\
\text { Protein }\end{array}$} & \multicolumn{4}{|c|}{ S. cerevisiae S288C } & \multirow[t]{2}{*}{ Predicted function } \\
\hline & & & & Homologue & Identity \% & $E$ value & Location $^{\mathrm{b}}$ & \\
\hline $1 s t$ & Trire2_120117 & $6.58 \times 10^{25}$ & TrCRE1 & Mig 1 & 72 & $7 e-30$ & Nucleus and cytoplasm & $\begin{array}{l}\text { Sequence-specific DNA } \\
\text { binding transcription factor } \\
\text { involved in the regulation of } \\
\text { transcription by RNA polymer- } \\
\text { ase II in response to glucose } \\
\text { and starvation }\end{array}$ \\
\hline 2nd & Trire2_120053 & $1.09 \times 10^{5}$ & TrHSP70 & Ssc1 & 74 & 0.0 & Mitochondrion & $\begin{array}{l}\text { Hsp70 family ATPase, involv- } \\
\text { ing in protein importing and } \\
\text { folding }\end{array}$ \\
\hline $3 r d$ & Trire2_121940 & $1.29 \times 10^{3}$ & TrTUP1 & Tup1 & 49 & $2 e-128$ & Nucleus & $\begin{array}{l}\text { General repressor of transcrip- } \\
\text { tion, forms complex with } \\
\text { Cyc } 8 p\end{array}$ \\
\hline 4th & Trire2_102616 & $3.82 \times 10^{2}$ & $\operatorname{TrCYC8}$ & Сус8 & 55 & $1 e-136$ & Nucleus & $\begin{array}{l}\text { General repressor of transcrip- } \\
\text { tion, forms complex with } \\
\text { Tup1p }\end{array}$ \\
\hline 5th & Trire2_122572 & $1.18 \times 10^{2}$ & - & Ssb2 & 73 & 0.0 & Cytoplasm and membrane & $\begin{array}{l}\text { Cytoplasmic ATPase that is a } \\
\text { ribosome-associated molecu- } \\
\text { lar chaperone }\end{array}$ \\
\hline 6th & Trire2_122920 & 6.31 & TrBIP1 & Kar2 & 76 & 0.0 & Endoplasmic reticulum & $\begin{array}{l}\text { ATPase involved in protein } \\
\text { import into the endoplasmic } \\
\text { reticulum }\end{array}$ \\
\hline 7th & Trire2_121906 & 4.01 & TrRPS14 & Rps14A & 84 & $6 e-63$ & Nucleus and cytoplasm & $\begin{array}{l}\text { mRNA-binding constituent of } \\
\text { the cytosolic small ribosomal } \\
\text { subunit; involved in matura- } \\
\text { tion of the small subunit rRNA } \\
\text { and assembly of the small } \\
\text { ribosomal subunit }\end{array}$ \\
\hline 8th & Trire2_119855 & 3.96 & TrRPS3 & Rps3 & 75 & $3 e-124$ & Cytoplasm & $40 \mathrm{~s}$ ribosomal protein \\
\hline 9th & Trire2_102864 & 3.64 & - & - & - & - & - & Uncharacterized protein \\
\hline 10th & Trire2_123753 & 2.98 & TrRPS31 & Rps31 & 85 & $8 e-67$ & Nucleus and cytoplasm & $\begin{array}{l}\text { Fusion protein that is cleaved } \\
\text { to yield ubiquitin and a } \\
\text { subunit of the cytosolic small } \\
\text { ribosomal subunit; involved } \\
\text { in maturation of the small } \\
\text { subunit rRNA, assembly of the } \\
\text { small ribosomal subunit, and } \\
\text { translation }\end{array}$ \\
\hline
\end{tabular}

-: No significant similarity found

a emPAl is the Exponentially Modified Protein Abundance Index of three samples. Every Peptide count of each sample is listed in Additional file 3: Spreadsheet S1

${ }^{b}$ Data from Saccharomyces Genome Database (www.yeastgenome.org)

In addition to PoCyc8 itself, 56 protein targets of putative interactions with $\mathrm{PoCyc} 8$ were captured (Additional file 3: Spreadsheet S1). The top 10 putative interacting protein targets with the highest emPAI are listed in Table 3. The top two proteins with the highest emPAI are PoCyc8 and PoTup1, thus verifying the stable interaction between PoTup 1 and PoCyc 8 and the consequent formation of the PoTup1-Cyc8 complex. Moreover, the 8th; 37th; 41st, and 45th positions are DNA-directed RNA Pol II subunit Rpb11; Rpb2 (the second largest subunit of Pol II); Rpb3 (the third largest subunit of Pol II), and Rpb1 (the largest subunit of Pol II), respectively (Additional file 3: Spreadsheet S1). PoCreA was also observed in the 50th position (Table 3, Additional file 3: Spreadsheet S1). This finding verified that PoCreA interacts with PoCyc8 in a direct or indirect way.

\section{PoCreA physically interacts with the PoCyc8-Tup 1 complex in the nucleus}

TAP-MS results for T. reesei TrCRE1 and P. oxalicum PoCreA suggested that CRE1/CreA recruits the Tup1Cyc8 complex. However, the putative interacting proteins identified by TAP-MS might include those that indirectly interact with CRE1/CreA as mediated by other proteins. In addition, the specific subunit of the complex that directly interacts with CRE1/CreA remains unknown. 
Table 2 The top 10 proteins interacting with PoCreA identified through TAP-MS

\begin{tabular}{|c|c|c|c|c|c|c|c|c|}
\hline \multirow[t]{2}{*}{ Rank } & \multirow[t]{2}{*}{ Gene locus } & \multirow[t]{2}{*}{ emPAl $^{a}$} & \multirow{2}{*}{$\begin{array}{l}\text { P. oxalicum 114-2 } \\
\text { Protein }\end{array}$} & \multicolumn{4}{|c|}{ S. cerevisiae S288C } & \multirow[t]{2}{*}{ Predicted function } \\
\hline & & & & Homologue & Identity \% & $E$ value & Location $^{\mathrm{b}}$ & \\
\hline $1 s t$ & PDE_03168 & $2.01 \times 10^{2}$ & PoCreA & Mig1 & 68 & $2 e-29$ & Nucleus and cytoplasm & $\begin{array}{l}\text { Sequence-specific DNA binding } \\
\text { transcription factor involved in } \\
\text { the regulation of transcription by } \\
\text { RNA polymerase II in response to } \\
\text { glucose and starvation }\end{array}$ \\
\hline 2nd & PDE_01024 & 11.18 & PoTup1 & Tup1 & 48 & $2 e-128$ & Nucleus & $\begin{array}{l}\text { General repressor of transcription, } \\
\text { forms complex with Cyc } 8 p\end{array}$ \\
\hline $3 r d$ & PDE_03177 & 2.16 & PoCyc8 & $\mathrm{Cyc8}$ & 58 & $7 e-149$ & Nucleus & $\begin{array}{l}\text { General repressor of transcription, } \\
\text { forms complex with Tup1p }\end{array}$ \\
\hline 4th & PDE_04157 & 1.22 & - & - & - & - & - & $\begin{array}{l}\text { Initiation-specific alpha-1,6-man- } \\
\text { nosyltransferase }\end{array}$ \\
\hline 5th & PDE_09900 & 0.74 & - & Thi13 & 66 & $3 e-175$ & Unknown & $\begin{array}{l}\text { Protein involved in synthesis of } \\
\text { the thiamine precursor HMP }\end{array}$ \\
\hline 6th & PDE_02746 & 0.50 & - & - & - & - & - & Putative protein \\
\hline 7th & PDE_09681 & 0.49 & - & Sps19 & 49 & $2 e-87$ & Peroxisome & $\begin{array}{l}\text { Peroxisomal 2,4-dienoyl-CoA } \\
\text { reductase involved in fatty acid } \\
\text { catabolism and sporulation }\end{array}$ \\
\hline 8th & PDE_07279 & 0.47 & - & Atp2 & 79 & 0.0 & Mitochondrion & $\begin{array}{l}\text { Subunit of the catalytic core of } \\
\text { the F1 sector of mitochondrial } \\
\text { F1FO ATP synthase }\end{array}$ \\
\hline 9th & PDE_04469 & 0.33 & - & Cct2 & 74 & 0.0 & Cytoplasm & $\begin{array}{l}\text { Subunit of the chaperonin- } \\
\text { containing T-complex (Tric) that } \\
\text { mediates protein folding }\end{array}$ \\
\hline 10th & PDE_03408 & 0.32 & - & Cdc19 & 66 & 0.0 & Cytoplasm & $\begin{array}{l}\text { Pyruvate kinase that catalyzes the } \\
\text { final step in glycolysis, the conver- } \\
\text { sion of phosphoenolpyruvate to } \\
\text { pyruvate, which is then utilized in } \\
\text { anaerobic or aerobic respiration }\end{array}$ \\
\hline
\end{tabular}

-: No significant similarity found

${ }^{a}$ emPAl is the Exponentially Modified Protein Abundance Index of three samples. Every Peptide count of each sample is listed in Additional file 3: Spreadsheet S1

${ }^{\mathrm{b}}$ Data from Saccharomyces Genome Database (www.yeastgenome.org)

Whether Tup1 or Cyc8 mediates the interaction between CRE1/CreA and the complex must be investigated.

BiFC analysis [34] was used to determine (1) the real physical interaction between PoCreA and PoTup1Cyc8 complex, and (2) the subunit of the complex that directly interacts with PoCreA. This method directly visualizes protein interactions in living cells. When two proteins gather together due to interaction, they carry two non-fluorescent fragments of yellow fluorescent protein (YFP) to complement each other, thus resulting in yellow fluorescence [35]. Several BiFC strains were constructed for the following analyses: PoCyc8-YFP-PoCreA strain to investigate the physical interaction between PoCreA and PoCyc8; PoTup1-YFPPoCreA strain to investigate the physical interaction between PoCreA and PoTup1; and PoCyc8-YFP-empty, PoTup1-YFP-empty, and empty-YFP-empty strains as a negative control. No significant difference in mycelia growth and conidia production was observed between the BiFC strains and the parent strain. In particular, their cellulolytic genes had a similar expression pattern (Additional file 2: Fig. S1C, D). The construction strategies and strain verification are shown in Additional file 4: Fig. S2.

TAP-MS results for PoCyc8 suggested that TF PoCreA recruits the complex by interacting with the subunit PoCyc8, however, its interaction with PoTup1 is unverified. Yellow fluorescence was observed in the nucleus of PoCyc8-YFP-PoCreA and PoTup1-YFPPoCreA BiFC strains (Fig. 1G, H) but not in any of the negative control BiFC strains (Additional file 2: Fig. S1E-G). These results suggest that PoCreA interacts with both PoTup1 and PoCyc8. The SWISS-MODEL SERVER [36] was then used to model PoTup1, PoCyc8, and PoCreA, respectively. The protein-protein docking between PoCreA and PoTup1-Cyc8 complex was predicted by the HDOCK SERVER [37]. The model with the highest score is shown in Additional file 5: Fig. S3. The putative model also supports the interaction of PoCreA with PoTup1 and PoCyc8. 
Table 3 The top 10 proteins interacting with PoCyc8 identified through TAP-MS

\begin{tabular}{|c|c|c|c|c|c|c|c|c|}
\hline \multirow[t]{2}{*}{ Rank } & \multirow[t]{2}{*}{ Gene locus } & \multirow[t]{2}{*}{ emPAl ${ }^{a}$} & \multirow{2}{*}{$\begin{array}{l}\text { P.oxalicum 114-2 } \\
\text { Protein }\end{array}$} & \multicolumn{4}{|c|}{ S. cerevisiae S288C } & \multirow[t]{2}{*}{ Predicted function } \\
\hline & & & & Homologue & Identity \% & $E$ value & Location $^{b}$ & \\
\hline $1 s t$ & PDE_03177 & $2.63 \times 10^{11}$ & PoCyc8 & Сус8 & 58 & $7 e-149$ & Nucleus & $\begin{array}{l}\text { General repressor of tran- } \\
\text { scription, forms complex } \\
\text { with Tup1p }\end{array}$ \\
\hline 2nd & PDE_01024 & $1.39 \times 10^{8}$ & PoTup1 & Tup1 & 48 & $2 e-128$ & Nucleus & $\begin{array}{l}\text { General repressor of tran- } \\
\text { scription, forms complex } \\
\text { with Cyc8p }\end{array}$ \\
\hline $3 r d$ & PDE_02075 & $3.16 \times 10^{3}$ & - & Nfs1 & 74 & 0.0 & $\begin{array}{l}\text { Nucleus and mitochon- } \\
\text { drion }\end{array}$ & $\begin{array}{l}\text { Mitochondrial cysteine } \\
\text { desulfurase involved in iron- } \\
\text { sulfur cluster assembly, tRNA } \\
\text { thio-modification and tRNA } \\
\text { wobble uridine modifica- } \\
\text { tion; subunit of L-cysteine } \\
\text { desulfurase complex }\end{array}$ \\
\hline 4 th & PDE_05635 & $1.92 \times 10^{2}$ & - & Rpl38 & 49 & $2 e-18$ & Cytoplasm and ribosome & $\begin{array}{l}\text { Subunit of the cytosolic } \\
\text { large ribosomal subunit; } \\
\text { involved in translation }\end{array}$ \\
\hline 5th & PDE_08686 & 18.14 & - & Vma2 & 85 & 0.0 & $\begin{array}{l}\text { Cytoplasm and vacuole } \\
\text { membrane }\end{array}$ & $\begin{array}{l}\text { Hydrogen ion transporting } \\
\text { ATPase involved in vacu- } \\
\text { olar acidification, calcium } \\
\text { homeostasis, and the assem- } \\
\text { bly of proteasome storage } \\
\text { granules }\end{array}$ \\
\hline 6th & PDE_05790 & 7.80 & - & Rpt6 & 77 & 0.0 & Nucleus and cytoplasm & $\begin{array}{l}\text { Putative ATPase involved } \\
\text { in proteasome regulatory } \\
\text { particle assembly }\end{array}$ \\
\hline 7th & PDE_01647 & 6.80 & - & Rpt1 & 77 & 0.0 & Nucleus and cytoplasm & $\begin{array}{l}\text { Putative ATPase involved } \\
\text { in proteasome regulatory } \\
\text { particle assembly }\end{array}$ \\
\hline 8th & PDE_00902 & 6.02 & - & Rpb11 & 50 & $4 e-38$ & Nucleus & $\begin{array}{l}\text { DNA-directed RNA polymer- } \\
\text { ase II subunit RPB11 }\end{array}$ \\
\hline 9th & PDE_08458 & 5.45 & - & Psa1 & 73 & 0.0 & Nucleus and cytoplasm & $\begin{array}{l}\text { Mannose-1-phosphate gua- } \\
\text { nyltransferase; synthesizes } \\
\text { GDP-mannose from GTP and } \\
\text { mannose-1-phosphate in } \\
\text { cell wall biosynthesis }\end{array}$ \\
\hline 10th & PDE_07279 & 5.39 & - & Atp2 & 79 & 0.0 & Mitochondrion & $\begin{array}{l}\text { Subunit of the catalytic core } \\
\text { of the F1 sector of mito- } \\
\text { chondrial F1F0 ATP synthase }\end{array}$ \\
\hline$\ldots \ldots$ & $\ldots \ldots$ & $\ldots \ldots$ & $\cdots \cdots$ & $\ldots \ldots$ & $\cdots \cdots$ & $\cdots \cdots$ & $\cdots \cdots$ & $\cdots \cdots$ \\
\hline 50th & PDE_03168 & 0.35 & PoCreA & Mig1 & 68 & $2 e-29$ & Nucleus and cytoplasm & $\begin{array}{l}\text { Sequence-specific DNA } \\
\text { binding transcription factor } \\
\text { involved in the regulation } \\
\text { of transcription by RNA } \\
\text { polymerase II in response to } \\
\text { glucose and starvation }\end{array}$ \\
\hline
\end{tabular}

a emPAl is the Exponentially Modified Protein Abundance Index of three samples. Every Peptide count of each sample is listed in the Additional file 3: Spreadsheet S1

${ }^{\mathrm{b}}$ Data from Saccharomyces Genome Database (www.yeastgenome.org)

-: No significant similarity found

PoCreA affected the histone methylation patterns of H3K4 and H3K36

TAP-MS and BiFC results revealed that CRE1/CreA physically interacts with Tup1-Cyc8 in the nucleus. Therefore, the mechanism on how CRE1/CreA-Tup1Cyc8 represses transcription must be determined. The initial hypothesis is that histone modification and chromatin structure change are the main mechanisms of the gene expression-inhibiting function of the complex [38]. Histone methylation, specifically on histone $\mathrm{H} 3$, regulates cellulolytic gene expression [39-42]. Even the expression of CRE1 itself is related to H3K4 methylation [43]. Whether CRE1/CreA-Tup1-Cyc8 is related to histone methylation and thus affects the transcription must 
be investigated. Two PocreA mutant strains including PocreA mutant $\triangle$ PocreA ( $\triangle$ PocreA::hph) [8] and PocreA mutant OEPocreA (ptrA::PgpdA::PocreA) [16] were chosen for the investigation of the effect of CRE1/CreATup1-Cyc8 interference on histone methylation.

First, the two mutants and the WT strain were cultivated on two different culture media: one is Vogel's minimal medium (VMM) plus glucose (VMMG), a medium that represses the expression of cellulase and hemicellulase encoding genes during catabolite repression initiated by glucose [44], and the other is VMM plus cellulose (VMMC), a medium that activates the expression of cellulase and hemicellulase encoding genes, as the degradation products of cellulose, such as small amounts of cello-oligosaccharides, act as inducers [45]. The same amount $\left(10^{6}\right)$ fresh spores of WT, $\triangle$ PocreA, and OEPocre $A$ were grown on VMMG or VMMC agar for 5 days. On VMMG agar, the $\triangle$ PocreA displayed diminished colony diameter. On VMMC agar, only the $\triangle$ PocreA mutant showed cellulolytic halo compared with the WT and OEPocreA mutant (Fig. 2A). This finding suggested that the $\triangle$ Pocre $A$ mutant secretes cellulolytic enzymes into the agar around the colony. The expression of two prominent cellulolytic genes, cellobiohydrolase-encoding gene Pocbh1 (cel7A, PDE_07945) and endoglucanase-encoding gene Poegl1 (cel7B, PDE_07929) was assayed after the strains were cultivated in VMMG liquid. The expression of gene Pocbh1 and gene Poegl1 was significantly upregulated in $\triangle$ PocreA but significantly downregulated in OEPocreA, in comparison to the WT (Fig. 2B). These results are consistent with previous reports and support the roles of PoCreA in CCR.

The mono-methylation (me1); di-methylation (me2), and tri-methylation (me3) of H3K4 (H3K4me1, H3K4me2, and H3K4me3) and H3K36 (H3K36me1, H3K36me2, and H3K36me3) and the mono-/di-methylation levels of H3K79 (H3K79me1 and H3K79me2) were also assayed when the strains were cultivated on in VMMG (Fig. 2C). $\triangle$ PocreA and OEPocreA mutants showed similar methylation patterns for H3K4me1, H3K36me1, H3K36me3, H3K79me1, and H3K79me2 compared with the WT. For the difference in the patterns of H3K4me3 and H3K36me2, the $\triangle$ PocreA mutant had a low level of H3K4me3 and no H3K36me2. Original Western blot images are shown in Additional file 6: Fig. S4.

Whether the dysregulation of PoCreA affects the expression of genes crucial for H3K4 and H3K36 methylation must be explored. Similar to $S$. cerevisiae having two methyltransferases Set1p and Set2p containing the evolutionarily conserved Su (var) 3-9, Enhancer-ofzeste, and Trithorax (SET) domain [46, 47], P. oxalicum also possesses two histone methyltransferase PoSet1 and PoSet2, which perform H3K4 and H3K36 methylation, respectively [40]. Therefore, the expression levels of Poset 1 and Poset 2 were investigated (Fig. 2D). The transcription of Poset 1 or Poset 2 did not show a difference (fold change $<2, P$ value $>0.05$ ) in either $\triangle$ Pocre $A$ mutant or OEPocreA mutant compared with that in the WT (Fig. 2D). This finding suggested that the effect of PoCreA dysregulation on the methylation patterns of $\mathrm{H} 3 \mathrm{~K} 4$ and H3K36 is not due to its influence on the transcription of Poset 1 or Poset2. The expression of gene Potup1 and Pocyc 8 was also assayed and did not show a significant difference (fold change $<2, P$ value $>0.05$ ) in the $\Delta$ Pocre $A$ mutant compared with that in the WT (Fig. 2E). This result suggested that the deletion of PocreA has no serious effect on the expression of these two genes. Pocyc8 gene was downregulated in the OEPocreA strain; however, the exact reason is unknown.

\section{PoCyc8 directly interacts with PoSet2, and the level of histone $\mathrm{H} 3 \mathrm{~K} 36 \mathrm{me} 2$ in the promoter of cellulolytic gene is positively correlated with PoCreA}

Saccharomyces Genome Database (SGD) verified that 51, 92, and 77 proteins physically interact with Mig1p, Tup1p, and Cyc8p, respectively. Among these proteins, histone methyltransferase Set2p physically interacts with Cyc8p [48]. Combined with the results of histone methylation patterns in Fig. $2 \mathrm{C}$, this finding gave a hint that the complex Tup1-Cyc8 is a bridge between the TF and the histone methyltransferase Set2. Therefore, the BiFC strain PoCyc8-YFP-PoSet2 was constructed to investigate the physical interaction between PoCyc8 and PoSet2. Analysis of the direct protein-protein interaction between PoCyc 8 and PoSet 2 revealed yellow fluorescence localized in the nucleus (Fig. 3A) but not in any of the negative control BiFC strains (Additional file 2: Fig. S1EG). This result indicated that the PoTup1-Cyc8 complex serves as a bridge for TF PoCreA and methyltransferase PoSet2.

Further analysis was conducted on the level of H3K36me2 in different regions upstream of the promoter of cellulase gene Pocbh1 and Poegl1 in mutants $\triangle$ Pocre $A$ and OEPocreA. The $5^{\prime}$ sequence of the promoters of Pocbh1 and Poegl1 was divided into three regions (Fig. 3B). The upstream of Pocbh1 promoter included region 1 ( -439 to -263$)$, region $2(-232$ to $-51)$, and region $3(-73$ to +95$)$. The upstream of Poegl1 promoter included region $1(-512$ to -344$)$, region $2(-316$ to -142$)$, and region $3(-155$ to +30$)$. Region 3 covers the eukaryotic core promoter, the minimal set of sequence elements required for accurate transcription initiation by the Pol II machinery [49]. Initiator (Inr) and the TATA boxes were found in the core promoter region for Pocbh1 and Poegl1 (Fig. 3B). Regions 1 and 2 were upstream of the core promoters 


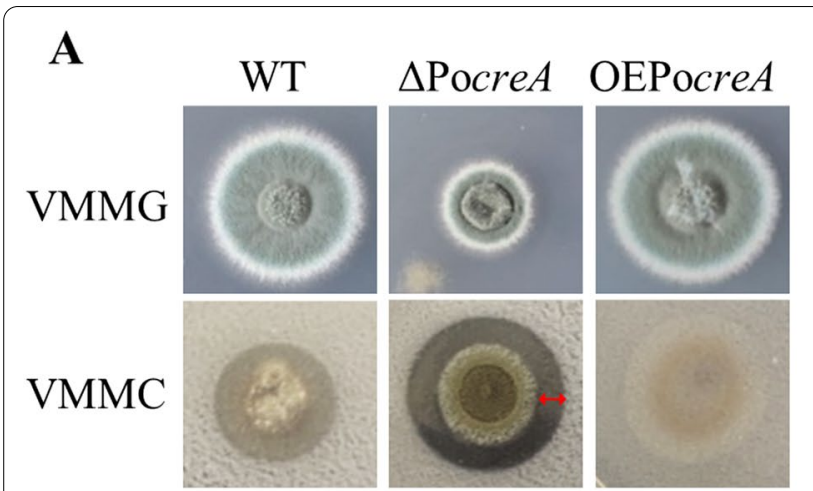

C

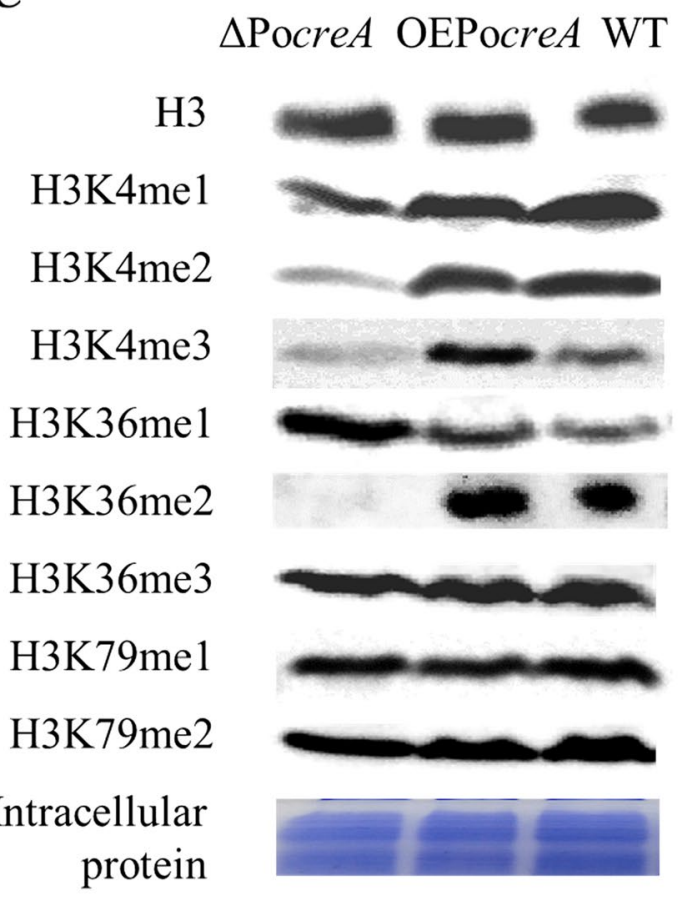

B

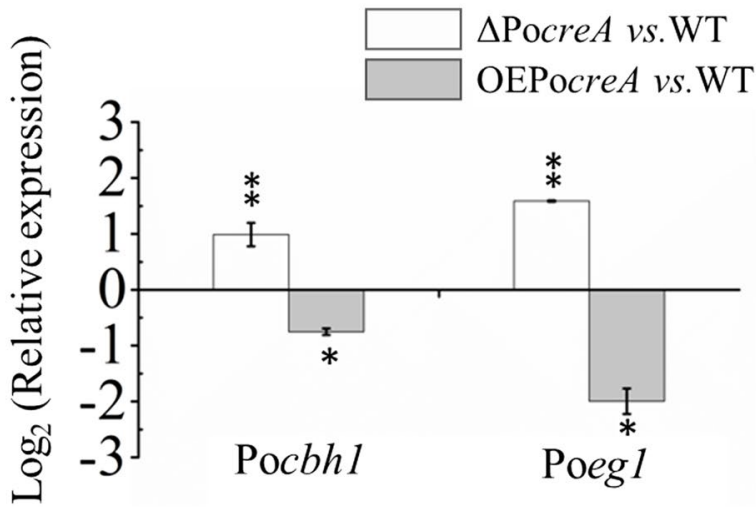

D

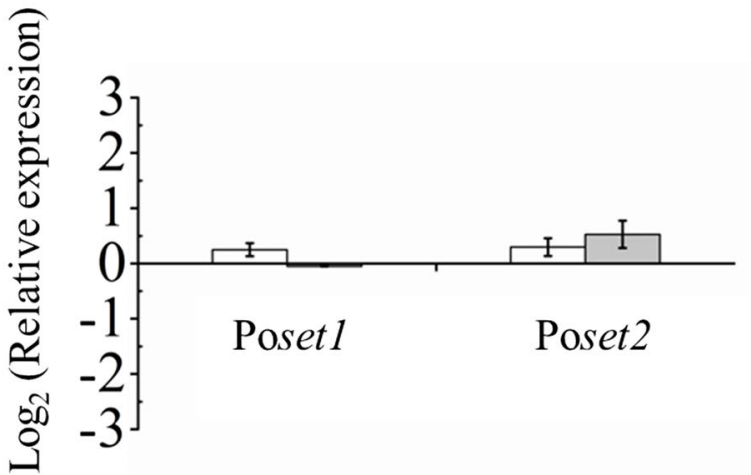

$\mathbf{E}$

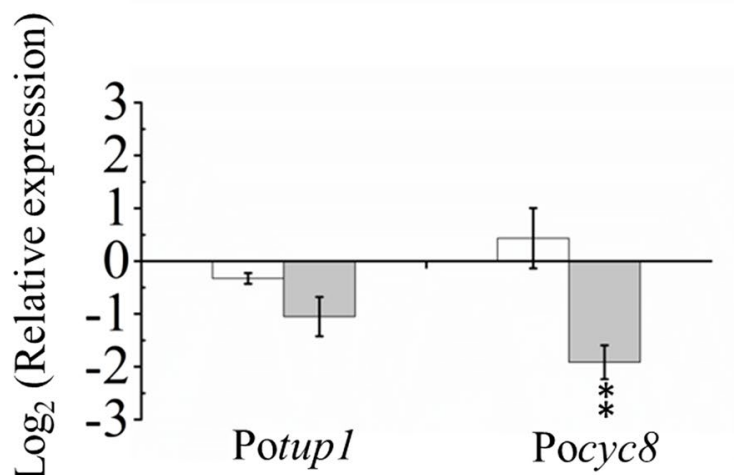

Fig. 2 Analysis of histone methylation patterns and transcription levels of genes in P. oxalicum WT and mutants. A Observation of cellulolytic halo around the colonies, red arrow represents cellulolytic halo. B The transcript abundance of two prominent cellulase encoding genes, Pocbh1 and Poeg/1. C Assays of histone methylation patterns using Western blot. Histone H3 was used as the loading control. D The transcript abundance of two histone methyltransferases encoding genes, Poset1 and Poset2. E The transcript abundance of genes Potup1 and Pocyc8. Statistical significance tests were performed by one tailed, unequal variance $t$-test. ${ }^{*} P<0.05,{ }^{* *} P<0.01,{ }^{* * *} P<0.001$

and contain the binding sequences for gene-specific IFs. The consensus sequence for PoCreA binding is 5'-SYGGRG-3' $[11,50]$. The Pocbh1 bores the putative PoCreA-binding sites 5'-GCGGAG-3' distributed in region -210 to -205 . The Poegl1 bores the putative PoCreA-binding sites 5'-CCGGGG-3', 5'-CCCCGC $-3^{\prime}, 5^{\prime}-\mathrm{CCCCGG}-3^{\prime}$, and 5'-CTCCGG-3' distributed in regions -77 to $-72,-237$ to $-232,-259$ to -254 , and -312 to -307 , respectively (Fig. 3B).
ChIP-qPCR is performed to analyze histone modifications of target loci in the genome. In ChIP-qPCR, immune-enriched DNA fragments are identified and quantified. ChIP was performed using anti-H3K36me2 antibody, combined with qPCR to detect the levels of histone H3K36me2 in the promoter of cellulolytic genes Pocbh1 and Poegl1. A remarkably increased level of H3K36me2 was observed in all the detected regions of Pocbh1 and Poegl1 for OEPocreA. In the $\triangle$ PocreA, 
a decreased level of H3K36me2 was observed in two (region 1 and region 3 ) of the three detected regions of Poegl1 but not in any region of Pocbh1 (Fig. 3C, D). The absence of PoCreA generally affected the low levels H3k36me2, but this influence was not significant. However, the overexpression of PocreA significantly increased the H3K36me2 level at specific cellulolytic gene loci. The reduced level of H3K36me2 in $\triangle$ PocreA and the increased level of H3K36me2 in OEPocreA implied that PoCreA is positively correlated with H3K36me2 level. In addition, the deletion of Poset2 ( $\triangle$ Poset2) [40], showed significantly decreased H3K36me2 levels in the three regions of Pocbh1 and Poegl1, indicating that H3K36me2 is mainly mediated by PoSet2 (Fig. 3C, D). Therefore, PoCreA recruits PoTup1-Cyc8. Histone methyltransferase PoSet 2 is also involved in the regulatory network via its interaction with PoCyc8. Given that PoSet2 and H3K36me2 on the promoter of cellulolytic genes are the repression marker of the target cellulolytic genes [40], the gene is inactivated by PoCreA-Tup1-Cyc8-Set2-mediated repression.

\section{Discussion}

CCR is a general phenomenon in various bacteria, yeast, filamentous fungi, and other microorganisms. The presence of carbon sources e.g., glucose and related sugars represses the transcription of certain genes. As a sequence-specific TF, CRE1/CreA plays a central role in CCR and is essential for the adaptation and survival of several species, such as Aspergillus, Penicillium, and Trichoderma [8, 13, 15]. In T. reesei, TrCRE1 rapidly shifts from cytoplasmic to nuclear with glucose addition [14] and represses the expression of glucose-repressible cellulolytic genes (such as cbh2 or egl1) [51]. Whether TrCRE1 binding ultimately regulates transcription upon DNA binding remains unclear.

In general, eukaryotic TFs regulate transcription without directly interacting with RNA Pol II but through recruiting cofactors that promote (or hinder) specific phases of transcription $[52,53]$. The cofactors might be "coactivators" or "corepressors"-usually large multisubunit protein complexes that regulate transcription via several different mechanisms. TAP-MS results for TrCRE1 and PoCreA, showed that as a homologue of yeast Mig1p, TrCRE1 and PoCreA might recruit the Tup1-Cyc8 complex involved in gene repression. These results verify the long-standing conjecture in the research field of filamentous fungi that CRE1/CreA recruits the corepressor complex Tup1-Cyc8 to participate in gene expression and CCR. In addition to CRE1/ CreA and Tup1-Cyc8, other regulators such as CreB, $\mathrm{CreC}$, and $\mathrm{CreD}$ also participate in CCR in A. nidulans [54]. However, the homologues of these proteins have not been identified from the putative interaction proteins of TrCRE1 or PoCreA. Alam et al. also reported the lack of direct physical interaction between CreA and CreB [54], which can be explained by two reasons. First, CreA does not directly interact with CreB. Second, the affinity of their direct interaction is low and was not detected due to the limitation of experimental technology.

More putative protein targets exhibit putative interactions with PoCyc8 than with PoCreA (Additional file 3: Spreadsheet S1). PoCyc8 and TrCYC8 are orthologs of S. cerevisiae $\mathrm{Cyc} 8$ and share $55 \%$ and $58 \%$ identity with the sequence of $S$. cerevisiae Cyc8, respectively. S. cerevisiae Cyc8p and PoCyc8 possess 10 copies of the 34-amino-acid tetratricopeptide repeat (TPR) motifs, and $\operatorname{TrCYC} 8$ possesses 9 copies of TPR (Additional file 7: Fig. S5A). TRP motifs form a helix-turn-helix arrangement and provide a structural scaffold for the mediation of multiple protein-protein interactions [55]. This finding explains the higher number of proteins interacting with the PoCyc8 than with PoCreA. In addition, the deletion of PoCyc8 is lethal in P. oxalicum, implying that the complex has more extensive regulatory roles than TF PoCreA.

The Tup1-Cyc8 complex is a conserved corepressor of transcriptional expression in eukaryotes. TrTUP1 and PoTup1 are orthologs of S. cerevisiae Tup1p, and share $49 \%$ and $48 \%$ identity with the sequence of $S$. cerevisiae Tup1, respectively. All of them possess seven highly conserved repeat WD40 domains (Additional file 7: Fig. S5B). These genes are single copy in T. reesei and P. oxalicum. In S. cerevisiae, the Tup1p-Cyc8

\footnotetext{
(See figure on next page.)

Fig. 3 Strategy and results of ChIP-qPCR. A Microscopy of PoCyc8-YFP-PoSet2 BiFC strain. The image includes four parts. The upper left, blue particles indicate nucleus stained with Hoechst 33342. The upper right, normal white light. The bottom right, yellow fluorescent particles indicate interactions between two PoCyc8 and PoSet2. The bottom left, indicating the merge of yellow fluorescence and blue nucleus. B Overview on the upstream sequence and core promoters of Pochb1 and Poeg/1. The transcription start site (TSS) is designated as + 1. The initiator (Inr) and TATA box were illustrated. The three chromatin regions investigated by ChIP-qPCR are indicated by green, red, and blue bars, respectively. For Pochb1, region 1 covers from -439 to -263 ; region 2 covers from -232 to -51 ; region 3 covers from -73 to +95 . For Poegl1, region 1 covers from -512 to -344; region 2 covers from -316 to -142 ; region 3 covers from -155 to +30 . The putative DNA-binding sites of PoCreA (5'-GCGGAG-3'; $5^{\prime}$-CCG GGG-3'; 5'-CCCCGC-3'; 5'-CCCCGG-3'; 5'-CTCCGG-3') are indicated by orange triangles. The orientation of the triangle represents the orientation of the binding motif. Inr, initiator element; TATA, TATA box. C ChIP-qPCR for H3K36me2 of Pocbh 1. D ChIP-qPCR for H3K36me2 of Poeg/1. All values are means from measurements in triplicates and three biological experiments. The error bars indicate standard deviations. ${ }^{*} P<0.05,{ }^{* *} P<0.01$, ${ }^{* * *} P<0.001$
} 


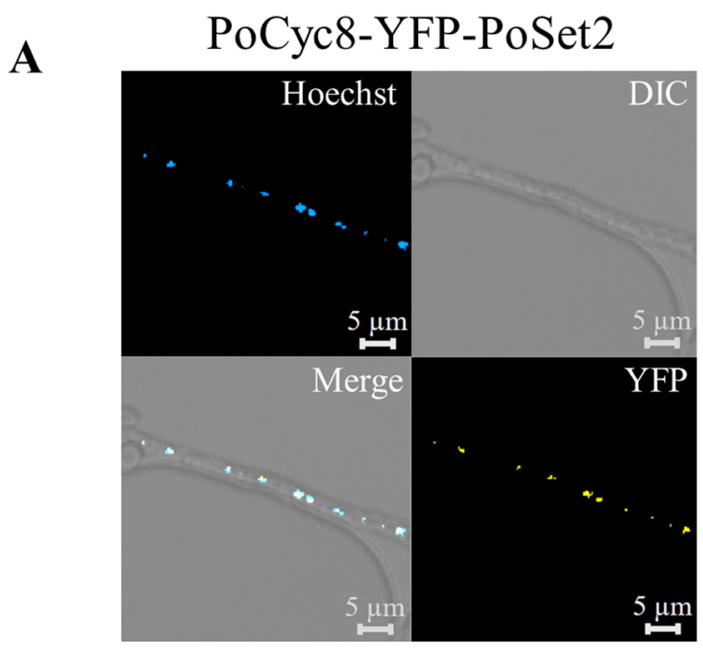

B
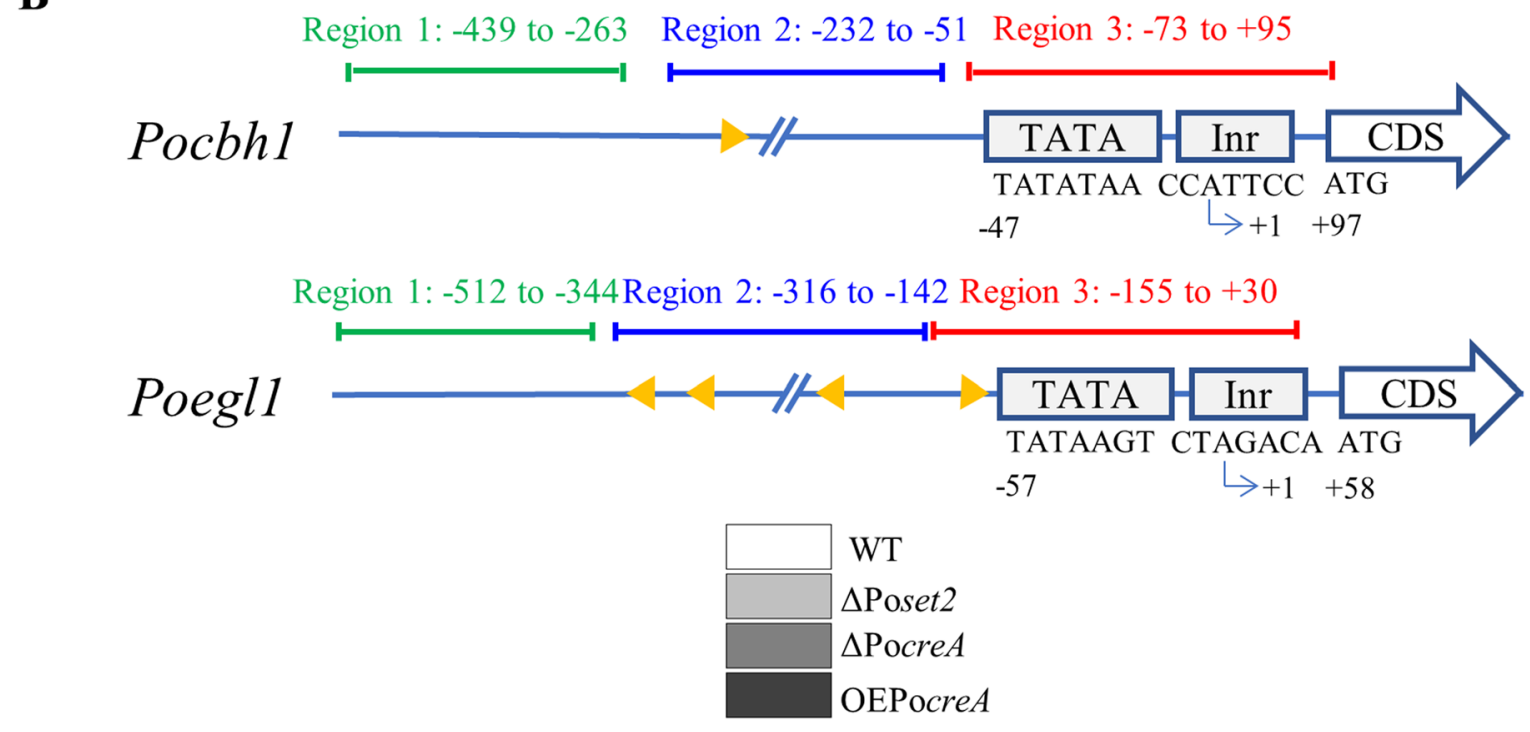

C

H3K36me2 (Pocbh1)

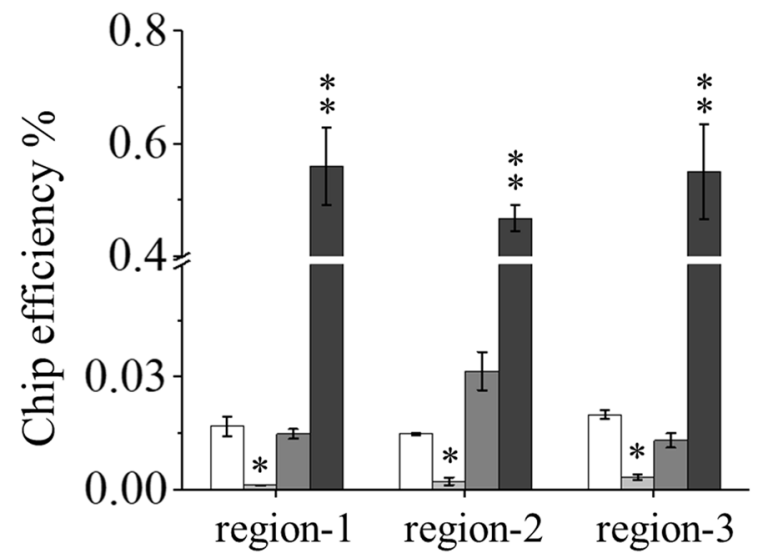

D $\mathrm{H} 3 \mathrm{~K} 36 \mathrm{me} 2(\mathrm{Poegl1})$

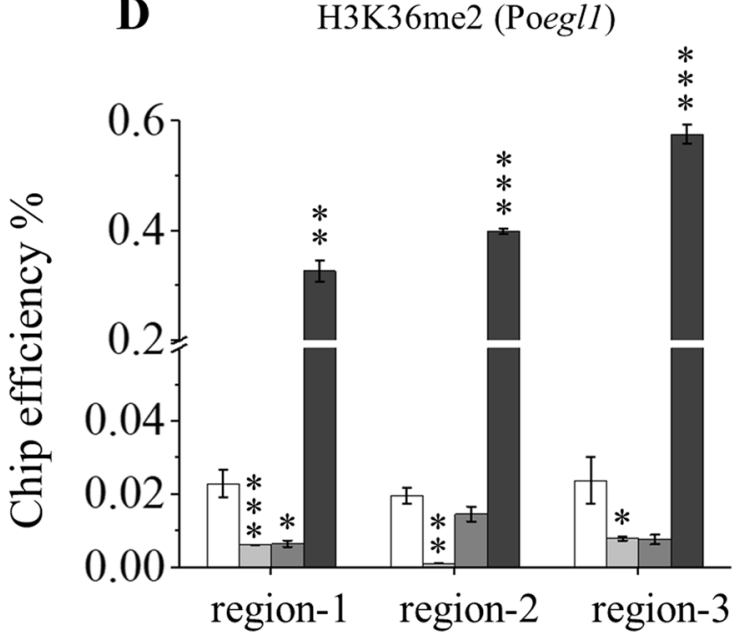

Fig. 3 (See legend on previous page.) 
complex is composed of four Tup1 and one Cyc8p subunit [56]. However, TAP-MS results for PoCyc8-TAP revealed that the emPAI of PoTup1 was lower than that of PoCyc8 (Table 3). Additional experimental evidence is needed to define the proportion of Tup 1 and Cyc 8 in the complex.

Although the mechanism for gene repression by the Tup1-Cyc8 complex in filamentous fungi remains poorly understood, several working models of Tup1-Cyc8 regulation in yeast have been proposed, including the interaction with histone deacetylases and modification of chromatin structures, interaction with the general transcription machinery, and blocking the activation domains of transcriptional activators [57]. This work showed that the mechanisms of turning genes off by PoCreA-Tup1Cyc8 in cellulolytic filamentous fungi exhibited similarities and differences with those in yeast.

Trichoderma reesei TrCRE1 was confirmed to be indirectly related to the change of chromatin structure, specifically on the promoter region of the cellulolytic genes; however, the exact reason is not known $[10,58]$. In $A$. nidulans, the deletion of the RcoA (the homologue of Tup1p) alters the chromatin structure of promoters for carbon catabolite repressible genes alcA, alcR, and prnD-prnB [27]. Therefore, CRE1/CreA-Tup1-Cyc8 might interact with some proteins related to chromatin modification, such as histone-modifying enzymes or chromatin-remodeling complexes. Direct interaction was observed between PoCyc8 and histone methyltransferase PoSet2, suggesting that the PoTup1-Cyc8 complex bridges the TF PoCreA and histone methyltransferase PoSet2. The transcription of Poset1 or Poset2 did not differ in either $\triangle$ PocreA mutant or OEPocreA mutant compared with that in the WT (Fig. 2D). Similar results were obtained from the analysis on the effects of CRE1/ CreA on the transcription of set1, set 2 , tup 1 , and $\operatorname{cyc} 8$ in other filamentous fungi according to their transcriptome data (Additional file 8: Fig. S6). Analysis was conducted on the data obtained for $T$. reesei $\operatorname{Tr}$ cre1 deletion strain (GEO accession: GSE57374) [5] and Magnaporthe grisea Mgcre1 deletion strain (GEO accession: GSE153084) [59] cultivated under glucose condition. In the cre1 deletion strains, the expression levels of set 1 gene (homologue ID 81925 in T. reesei and MGG_15053 in M. grisea), set2 gene (homologue ID 80732 in T. reesei and MGG_01661 in M. grisea), tup1 gene (homologue ID 121940 in T. reesei and MGG_08829 in M. grisea), and $c y c 8$ gene (homologue ID 102616 in T. reesei and MGG_03196 in $M$. grisea) in the mutants were not different from those of their corresponding parent strains, with the fold changes for transcripts $<2$ (Additional file 8: Fig. S6). These results suggested that CRE1/CreA does not directly affect the expression of the above genes. P. oxalicum PoCreA possibly affects histone methylation through other mechanisms.

In $S$. cerevisiae, Set2p physically interacts with Cyc8p [48]. Although the deletion of Set2p in yeast does not affect the Tup1-Cyc8-mediated repression of well-defined targets [60], our previous study showed that the deletion of $P$. oxalicum Poset 2 upregulated the transcription of cellulolytic genes accompanied by a decrease in H3K36 methylation on specific cellulolytic gene loci [40]. Meanwhile, Poset 2 overexpression downregulated the transcription of cellulolytic genes accompanied by changes in the chromatin structure around the promoter and transcription start site (TSS) [40]. In the present study, the level of histone H3K36me2 in the promoter of cellulolytic genes was found to be positively correlated with PoCreA protein levels. Therefore, a high amount of PoCreA protein presumably recruits a high amount of PoTup1-Cyc8 complex and PoSet2, followed by a high level of methylation of H3K36, a change in the local chromatin environment, and repressed cellulolytic genes.

In the regulation of PoCreA-Tup1-Cyc8-Set2, H3K36 methylation was discovered as a repression marker for cellulolytic gene transcription. This result is unexpected because Set $2 p$ is commonly associated with transcriptional activation [46, 61]. However, many reports supported the important role of Set2p in gene repression. For example, approximately 80 mRNA genes in yeast were activated upon Set2p absence [62]. Set2p also prevents transcription initiation by recruiting a repressive histone deacetylase (HDAC) Rpd3S complex to change the chromatin structures after Pol II passage, thereby suppressing transcription initiation and slowing down elongation [38, 63]. Meanwhile, yeast Tup1p-Cyc8p repression functions are always linked to the changes in chromatin structure mediated by recruiting Rpd3S complex [64], which supports the relation between the complex Tup $1 p-C y c 8 p$ and Set2p.

In yeast, the gene repression effect of corepressor complex Tup1-Cyc8 is also related to the general transcription machinery. Once at the promoter, the complex Tup1-Cyc8(Ssn6) interacts with mediator subunits, such as SIN4/MED16, Hrs1/MED3, and SRB7/MED21, thus preventing DNA-directed Pol II holoenzyme to be recruited to the core promoter or halt transcription initiation $[65,66]$. However, no mediator subunit was found in the results of PoCyc8TAP, although the PoTup1-Cyc8 complex was evident. Four Pol II subunits, namely, Rpb1, Rpb2, Rpb3, and Rpb11 were observed. Rpb1 and Rpb2, as the largest and second-largest catalytic subunits of RNA Pol II, together with third-largest subunit Rpb3, and Rpb10, Rpb11, Rpb12 subunits, form the central large cleft, which is the polymerase active center [67]. Yeast Cyc8p 
also directly interacts with Rpb3p as revealed by Affinity Capture-Western assay [68]. Therefore, another hypothesis for these results is that PoCreA-Cyc8-Tup1mediated repression occurs via direct interaction with some components of the Pol II (possibly subunit Rpb3) and hinders the Pol II from progressing downstream of the promoter. However, it would necessitate further research to confirm this hypothesis.

Although no direct evidence confirms that TF CRE1/ CreA, complex Tup1-Cyc8, RNA Pol II and histone methyltransferase Set 2 co-occupy on the promoter, a new research in yeast supported that Tup1p, RNA Pol II (Rpb3p), and Set2p occupy near the TSS [69]. On the basis of previous reports and present data, a model for PoCreA-Tup1-Cyc8 during the repression of the cellulolytic gene was proposed (Fig. 4). In the presence of glucose, PoCreA mainly localizes in the nucleus, binds to the promoter of the target genes, and recruits the corepressor complex PoTup1-Cyc8. Histone methyltransferase Set2, which methylates H3K36, is also involved in the regulatory network by interacting with PoCyc8. As the repression marker of cellulolytic gene expression, H3K36 methylation and histone deacetylase Rpd3 cooperate to reestablish chromatin, thereby suppressing inappropriate transcription initiation. In addition, the corepressor PoTup1-Cyc8 also interacts with the main subunit of the RNA Pol II and thus prevents Pol II from initiating transcription (Fig. 4). It is noting the model is mainly applicable to $P$. oxalicum, although the interaction between CRE1/CreA and co-repressor complex Cyc8-Tup1 is conservative.

\section{Conclusions}

This study verifies the long-standing conjecture that TF CRE1/CreA represses gene expression via interacting with the corepressor complex Tup1-Cyc8 in two cellulase-producers T. reesei and P. oxalicum. An explanation that the cellulolytic gene is repressed by PoCreATup1-Cyc8-Set2-mediated transcriptional repression in $P$. oxalicum, was presented. The findings contribute to the understanding of CCR mechanism in filamentous fungi and serve as a guide for biotechnologically relevant enzyme production.

\section{Material and methods}

\section{Strains and culture condition}

The WT strain P. oxalicum 114-2 (CGMCC 5302) and the mutants $\triangle$ PocreA and OEPocreA $[8,16]$ were cultivated on $10 \%$ wheat bran extract agar slants at $30{ }^{\circ} \mathrm{C}$ for 5 days. T. reesei QP4 [31] was cultivated on potato dextrose agar (PDA) with Vogel's minimal medium (VMM) [70] agar added with $2 \%$ glucose (VMMG) at $30{ }^{\circ} \mathrm{C}$ for 5 days. All strains used in this study are listed in Additional file 1: Table S1.

\section{Construction of strains for TAP and BiFC}

For TAP strains, the homologous recombination was used to knock-in FLAG and HA tags before the C-terminal stop codon of the bait protein. The strategy of TAP strains construction is shown in Additional file 4: Fig. S2A. To construct the TrCRE1-TAP strain (TrCRE1FLAG-HA) in T. reesei QP4: primers TrCRE1-F/TrCRE1tap- $\mathrm{R}$ were used to amplify the upstream homologous

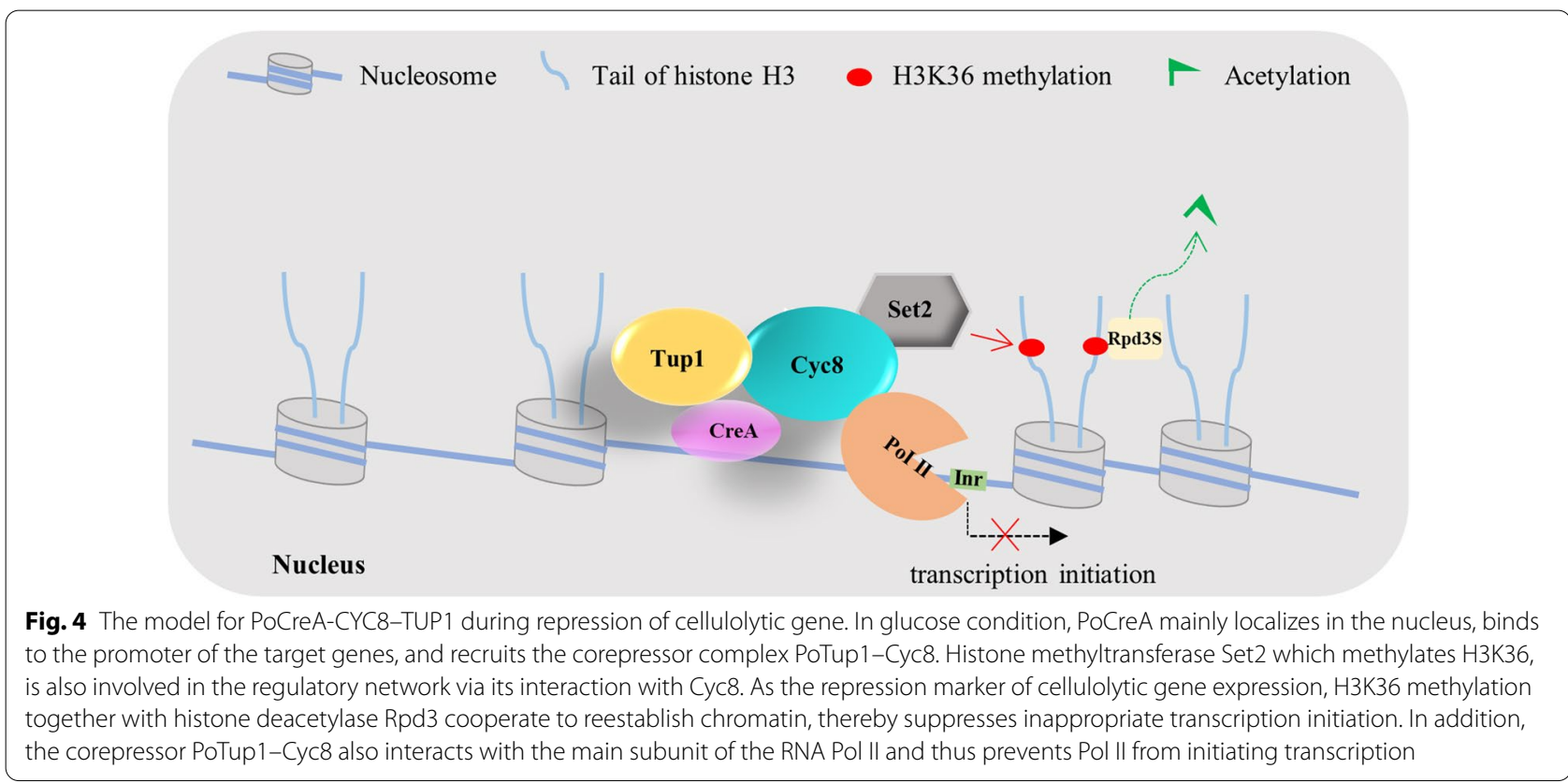


arm (1585 bp) of the gene Trcre1. Primers TrCRE1-DF/ TrCRE1-DR were used to amplify the downstream homologous arm (1667 bp) of the gene Trcre1. Primers pyrG-F/pyrG-R were used to amplify the marker gene pyrG (1434 bp) from the genome of $A$. nidulans. The upstream homologous arm, pyrG gene, and downstream homologous arm were fused by overlapping PCR and then amplified by nested primers TrCRE1-CSF/TrCRE1CSR. The fused PCR product (4464 bp) was transformed into T. reesei QP4 through polyethylene glycol (PEG)mediated protoplast transformation [71] to obtain the TAP strain TrCRE1-TAP. The same method was applied to construct $P$. oxalicum PoCreA-TAP strain (PoCreAFLAG-HA), and PoCyc8-TAP strain (PoCyc8-FLAGHA). Primers PoCreA-F/PoCreA-tap-R and PoCyc8-F/ PoCyc8-tap-R were used to amplify the upstream homologous arms of the gene PocreA and Pocyc8 (2109 and 3466 bp, respectively). Primers PoCreA-DF/PoCreADR and PoCyc8-DF/PoCyc8-DR were used to amplify the downstream homologous arms of gene PocreA and Pocyc8 (1998 and 1521 bp, respectively). Primers hygAF/hygA-R were used to amplify the marker hygromycin gene hygA (1954 bp) from the template of plasmid pSilent1 [72]. The upstream homologous sequence, hygA gene and downstream homologous sequence were fused by overlapping PCR and then amplified by nested primers PoCreA-CSF/PoCreA-CSR and PoCyc8-CSF/PoCyc8CSR, respectively. The two fused PCR products (5275 and $6752 \mathrm{bp}$ ) were transformed into $P$. oxalicum 114-2 using PEG-mediated protoplast transformation [71] to obtain the TAP strains PoCreA-TAP, and PoCyc8-TAP, respectively. The primers used for PCR amplification are listed in Additional file 9: Table S2.

BiFC strains were constructed as previously described [34], and the strategy of construction is shown in Additional file 4: Fig. S2. The plasmid pMD18-T-NYFP carries the encoding sequence of $\mathrm{N}$-terminal (1-155 aa) of the yellow fluorescent protein (YFP) (Additional file 4: Fig. S2C), and the plasmid pUC19-CYFP carries the encoding sequence of C-terminal (156-238 aa) of the YFP (Additional file 4: Fig. S2D). Primers PoTup1-NF/ PoTup1-NR and PoCyc8-NF/PoCyc8-NR were used to amplify the Potup 1 and Pocyc8 genes, respectively, which were inserted into the multiple cloning site (MCS) of pMD18-T-NYFP to obtain the recombined pMD18-TNYFP-PoTup1 and pMD18-T-NYFP-PoCyc8 vectors, respectively. Similarly, the primers PoCreA-CF/PoCreA$\mathrm{CR}$ and PoSet2-CF/PoSet2-CR were used to amplify the genes PocreA and Poset2, which were then inserted into the MCS of pUC19-CYFP to obtain the recombined pUC19-CYFP-PoCreA and pUC19-CYFP-PoSet2 vectors, respectively. Vectors pMD18-T-NYFP-PoTup1 and pUC19-CYFP-PoCreA were simultaneously transformed into the parent strain 114-2 to study the interaction between PoTup1 and PoCreA. Vectors pMD18-T-NYFPPoCyc8 and pUC19-CYFP-PoCreA were simultaneously transformed into the parent strain 114-2 to study the interaction between PoCyc8 and PoCreA. Vectors pMD18-T-NYFP-PoCyc8 and pUC19-CYFP-PoSet2 were simultaneously transformed into the parent strain 114-2 to study the interaction between PoCyc8 and PoSet2. Similar method was used to construct negative control strains, namely, PoTup1-YFP-empty, PoCyc8-YFP-empty, and empty-YFP-empty. Two pairs of primers NYZF/ NYZR and CYCF/CYZR were used to verify the BiFC strains (Additional file 4: Fig. S2E). The primers used for PCR amplification are listed in Additional file 9: Table S2.

\section{Phenotypic analysis and enzyme activity determination}

For phenotype analysis, the fresh spore suspension was diluted to the same concentration $\left(10^{6}\right.$ conidia $\left./ \mathrm{mL}\right) .1 \mu \mathrm{L}$ of spore suspension were spotted on VMMG agar at $30{ }^{\circ} \mathrm{C}$ for 5 days. For enzyme activity assay, fresh spore suspensions of the parent and mutant strains were cultivated in VMMG liquid for $24 \mathrm{~h}$. Afterward, $0.3 \mathrm{~g}$ of filtered hyphae was transferred to $100 \mathrm{~mL}$ of $\mathrm{VMM}$ added with $1 \%$ bran juice and $1 \%$ cellulose $(\mathrm{w} / \mathrm{v})$ media and mixed at $180 \mathrm{rpm}$ and $30{ }^{\circ} \mathrm{C}$. The filter paper enzyme activities (FPA) of the culture supernatants were assayed using DNS reagent [73]. Whatman No. 1 filter paper (GE Healthcare companies, UK) was applied as the substrate. One enzyme activity unit is defined as the amount of enzyme that can convert $1 \mu \mathrm{mol}$ of the substrate in $1 \mathrm{~min}$ under the assay conditions.

\section{Microscopy of BiFC strains}

Fresh spore suspensions of BiFC strains PoTup1-YFPPoCreA; PoCyc8-YFP-PoCreA; PoCyc8-YFP-PoSet2 and negative control strains PoTup1-YFP-empty; PoCyc8YFP-empty; empty-YFP-empty were spread on VMMG agar. Then, $18 \mathrm{~mm}$ sterile coverslips were inserted into the agar at a $45^{\circ}$ angle. The cultures were incubated at $30{ }^{\circ} \mathrm{C}$ for $24 \mathrm{~h}$. Hoechst 33342 (Sigma-Aldrich, United States) was used for nucleus staining. The blue nucleus stained by Hoechst 33342 was observed under $405 \mathrm{~nm}$ excitation light. Yellow fluorescence was observed by excitation light at $488 \mathrm{~nm}$ using the laser scanning confocal microscope (ZEISS LSM900) (Carl Zeiss).

\section{Protein-protein docking and domain architecture analysis} The SWISS-MODEL SERVE [36] was used to model target proteins. The 3D protein model was automatically generated by inputting the amino acid sequence of the target protein. The highest-scoring protein models of PoCreA, PoCyc8, and PoTup1 were individually created. The HDOCK SERVER [37] was then used to predict 
the protein-protein docking model. First, the models of PoCyc8 and PoTup1 were inputted as the receptor and ligand, respectively, to obtain the highest-scoring PoTup1/Cyc8 docking model. The models of PoTup1/ $\mathrm{Cyc} 8$ and PoCreA were then inputted as the receptor and ligand, respectively, to obtain the PoCreA-Tup1/Cyc8 docking model.

\section{Total RNA extraction and gene expression analysis by qRT-PCR}

The fresh spore suspensions of parent strain 114-2 and mutants $\triangle$ PocreA and OEPocreA were cultivated in VMMG liquid for $24 \mathrm{~h}$. The mycelia were collected and ground in liquid nitrogen, and $100 \mathrm{mg}$ of ground powder was then transferred into $1 \mathrm{~mL}$ of TRIzol reagent (TaKaRa Biotechnology). Total RNA extraction was performed in accordance with the manufacturer's instructions. cDNA was obtained by PrimeScript RT Reagent kit with gDNA Eraser (TaKaRa Biotechnology). Three biological triplicates of qPCR assay of each gene were performed. Light Cycler 480 system with software version 4.0 (Roche, Mannheim, Germany) was used to perform the reaction procedure. The primers of expression of the specific gene Pocbh1, Poegl1, Poset1, Poset2, Potup1, Pocyc8, and Poactin assayed by qPCR are as follows: qPocbh1F/qPocbh1R; qPoegl1F/qPoegl1R; qPoset1F/ qPoset1R; qPoset2F/qPoset2R; qPotup1F/qPotup1R; qPocyc8F/qPocyc8R; and qPoactinF/qPoactinR. The expression level of a specific gene is based on the control gene Poactin (PDE_01092). The outcome of relative expression of the examined gene was calculated as follows: copy number of target gene/actin gene. Statistical significance was considered at $P \leq 0.05$. The primers used for qPCR are listed in Additional file 9: Table S2.

\section{Protein extraction and Western blot analysis}

The fresh spore suspensions of parent strain 114-2 and mutants $\triangle$ PocreA and OEPocreA were cultivated in VMMG liquid for $24 \mathrm{~h}$. The mycelia were collected and ground in liquid nitrogen, and $100 \mathrm{mg}$ of ground powder was transferred into $200 \mu \mathrm{L}$ of extraction buffer (per liter: $1 \mathrm{M} \mathrm{pH7.5} \mathrm{Tris-HCl} 50$ mL, NaCl 8.76 g, NP-40 $10 \mathrm{~mL}$, $100 \mathrm{mM}$ phenylmethanesulfonyl fluoride (PMSF) $10 \mathrm{~mL}$ ). The samples were vigorously mixed by a vortex shaker, placed in an ice bath for $30 \mathrm{~min}$, and centrifuged at $4{ }^{\circ} \mathrm{C}$, $12,000 \mathrm{rpm}$ for $10 \mathrm{~min}$ to obtain the supernatant. Protein concentrations in the supernatant were assayed using the Bradford method [74]. Equal amounts (200 ng) of total protein were separated by SDS polyacrylamide gel electrophoresis (SDS-PAGE) and then transferred to nitrocellulose membrane (Pall Corp., Ann Arbor, MI, United States) using a Bio-Rad electroblotting apparatus. The anti-H3K4me1 antibody (ab8895, Abcam, Cambridge,
UK), anti-H3K4me2 antibody (A2356, ABclonal, Wuhan, China), and anti-H3K4me3 antibody (ab8580, Abcam, Cambridge, UK) were used to detect H3K4 methylation. The anti-H3K36me1 antibody (OM256826, OmnimAbs, California, USA), anti-H3K36me2 antibody (A2365, ABclonal, Wuhan, China), and anti-H3K36me3 antibody (ab9050, Abcam, Cambridge, UK) were used to detect H3K36 methylation. The anti-H3K79me1 antibody (OM256854, OmnimAbs, California, USA) and antiH3K79me2 antibody (ab3594, Abcam, United Kingdom) were used to detect H3K79 methylation. Equal amounts of the total protein and the anti-histone $\mathrm{H} 3$ antibody (OM256785, OmnimAbs, California, USA) were set as the loading control. Western blot was performed following the detailed method described in [40].

\section{Chromatin immunoprecipitation and qRT-PCR (ChIP-qPCR) assay}

Fresh spore suspensions of parent strain 114-2 and mutants $\triangle$ PocreA and OEPocreA were inoculated in VMMG liquid for $24 \mathrm{~h}$ and then added with $37 \%$ formaldehyde to crosslink the samples for $10 \mathrm{~min}$ and finally with $1.25 \mathrm{M}$ glycine to terminate the crosslink procedure. Pre-cooled TBS buffer was used to wash the mycelia, which were then drained and ground with liquid nitrogen. An appropriate amount of Chip-lysis buffer was added to lyse the ground mycelia to obtain the supernatant through centrifugation. The supernatant was separated through sonication with the condition of $10 \mathrm{~s}$ on and $10 \mathrm{~s}$ off for 72 cycles on ice to ensure that the chromatin was broken to $100-1000 \mathrm{bp}$. Afterward, $20 \mu \mathrm{L}$ of blocked protein G/A beads (Thermo Fisher Scientific, MA, United States) were added in per $1.1 \mathrm{~mL}$ of the disrupted solution and stored in $4{ }^{\circ} \mathrm{C}$ for $4 \mathrm{~h}$. In brief, $100 \mu \mathrm{L}$ of the sample was obtained, labeled as input, and added with $1 \mu \mathrm{L}$ of anti-H3K36me 2 antibody to react overnight. Afterward, $50 \mu \mathrm{L}$ of protein G/A beads were added to incubate for $4 \mathrm{~h}$. Finally, the beads were eluted and de-crosslinked overnight with $20 \mu \mathrm{L}$ of $5 \mathrm{M} \mathrm{NaCl}$ at $65^{\circ} \mathrm{C}$. DNA was extracted with phenol/chloroform/isoamyl alcohol. ChIP-enriched genomic DNA fragments were assayed by qPCR analysis using the following primers: Pocbh1-1F/ Pocbh1-1R; Pocbh1-2F/Pocbh1-2R; Pocbh1-3F/Pocbh13R, and Poegl1-1F/Poegl1-1R; Poegl1-2F/Poegl1-2R; Poegl1-3F/Poegl1-3R. The relative enrichment of IP DNA was calculated by the input \% method as follows $(\mathrm{Ct}=$ the number of cycles required to reach the threshold): ChIP efficiency $=2^{-\Delta \mathrm{Ct}} \times 100 \%, \quad \Delta \mathrm{Ct}=\mathrm{Ct}_{\mathrm{IP}}-\left(\mathrm{Ct}_{\text {Input }}-\log _{2} 10\right)$ [40]. Three biological replicate experiments were performed for each strain. Statistical significance was considered at $P \leq 0.05$. The primers used for ChIP-qPCR are listed in Additional file 9: Table S2. 


\section{TAP and mass spectrometry}

Fresh spore suspensions of the parent strain $P$. oxalicum 114-2, PoCreA-TAP, PoCyc8-TAP, T. reesei QP4 and TrCRE1-TAP strain were inoculated in $2 \mathrm{~L}$ of VMM liquid added with $2 \%$ glucose (VMMG) as a carbon source at $180 \mathrm{rpm}$ for $24 \mathrm{~h}$, at $30{ }^{\circ} \mathrm{C}$ in a shaker. The hyphae were filtered and washed by distilled water twice, ground with liquid nitrogen, transferred to a $100 \mathrm{~mL}$ centrifuge tube, and added with $15 \mathrm{~mL}$ of protein lysis buffer $(\mathrm{NaCl} 9 \mathrm{~g}$, $1 \mathrm{M}$ Tris- $\mathrm{HCl}, \mathrm{pH} 7.5$, glycerin $100 \mathrm{~mL}$, and NP40 $1 \mathrm{~mL}$, per $1 \mathrm{~L}$ ) and $0.05 \%$ protease inhibitor cocktail. The samples were then centrifuged at $12,000 \mathrm{rpm}$ and $4{ }^{\circ} \mathrm{C}$ for $30 \mathrm{~min}$ to obtain the suspension. For the first-step affinity purification, ANTI-FLAG M2 affinity resin (SigmaAldrich, United States) was added to the suspension and incubated overnight at $4{ }^{\circ} \mathrm{C}$ with rotation. The protein suspension was then centrifuged at $3000 \mathrm{rpm}$ for $2 \mathrm{~min}$ at $4{ }^{\circ} \mathrm{C}$ to discard the supernatant. ANTI-FLAG M2 affinity resin was transferred to the spin columns and centrifuged at $3000 \mathrm{rpm}$ for $30 \mathrm{~s}$ at $4{ }^{\circ} \mathrm{C}$ to discard the filtrate. Afterward, $500 \mu \mathrm{L}$ of $3 \times$ FLAG peptide (final concentration $150 \mathrm{ng} / \mu \mathrm{L}$ ) (Sigma-Aldrich, United States) was added to the spin columns and centrifuged at $3000 \mathrm{rpm}$ for $1 \mathrm{~min}$ to obtain the first-step eluent. For the secondstep affinity purification, the ANTI-HA resin (Thermo Fisher Scientific, MA, United States) was transferred to the first-step eluent, incubated at $4{ }^{\circ} \mathrm{C}$ for $2 \mathrm{~h}$, transferred to the spin columns, and centrifuged at $3000 \mathrm{rpm}$ for $30 \mathrm{~s}$ at $4{ }^{\circ} \mathrm{C}$ to discard the filtrate. Finally, $80 \mu \mathrm{L}$ of $8 \mathrm{M}$ urea was added and incubated with the ANTI-HA resin for $15 \mathrm{~min}$. The spin columns were centrifuged at $3000 \mathrm{rpm}$ for $1 \mathrm{~min}$ to obtain the final eluent, which was then divided into three parts: one for Western blot using the ANTI-HA antibody (ABclonal, China), one was separated by $12.5 \%$ SDS-PAGE and stained with silver reagent [75], and the last one was assayed through LC-MS/MS (APT, Shanghai, China) to determine the putative interacting proteins from the bait proteins. Exponentially modified protein abundance index (emPAI) was used for estimation of absolute protein amount using the following formula. Where $N_{\text {observed }}$ is the number of experimentally observed peptides, and $N_{\text {observable }}$ is the number of theoretically observable tryptic peptides for each protein. $\mathrm{PAI}=N_{\text {observed }} / N_{\text {observable, }}$ emPAI $=10^{\mathrm{PAI}}-1$ [32].

\section{Supplementary Information}

The online version contains supplementary material available at https://doi. org/10.1186/s13068-021-02092-9.

Additional file 1: Table S1. The strains used in this study.

Additional file 2: Figure S1. Phenotypic analysis and enzyme activity determination of TAP and BiFC strains. (A) Growth phenotype of TrCRE1TAP strain and parent T. reesei QP4. (B) FPA activities assay of TrCRE1-TAP strain and parent T. reesei QP4. (C) Phenotypic analysis of TAP and BiFC strains in P. oxalicum. (D) FPA activities assay of TAP and BiFC strains in P. oxalicum. (E) Microscopy of PoTup1-YFP-empty BiFC strain. (F) Microscopy of PoCyc8-YFP-empty BiFC strain. (G) Microscopy of empty-YFP-empty BiFC strain.

Additional file 3: Spreadsheet S1. Proteins interacting with T. reesei TrCRE1, P. oxalicum PoCreA, and PoCyc8 identified through TAP-MS. The peptide counts (PepCount) of each biological replicate, the sum of PepCount of three biological replicates, the number of observable peptides, and Exponentially Modified Protein Abundance Index (emPAl) were listed. The proteins are arranged according to the value of emPAI.

Additional file 4: Figure S2. Construction strategy and verification of TAP and BiFC strains. (A) Construction strategy of TAP strains. (B) Results of diagnostic PCR of TAP strains. Lane 1 (1895 bp) and Lane 2 (1928 bp) represent TrCRE1-TAP (amplified using primers TrCRE1-F/pyrG-YZR and pyrGYZF/TrCRE1-DR, respectively); lane 3 and Lane 4 represent negative contro (T. reesei QP4); lane 5 (2289 bp) and Lane 6 (2234 bp) represent PoCreA-TAP (amplified using primers PoCreA-F/hygA-YZR and hygA-YZF/PoCreA-DR respectively); lane 7 and lane 8 represent negative control (P. oxalicum 114-2); lane 9 (3646 bp) and lane 10 represent PoCyc8-TAP (amplified using primers PoCyc8-F/hygA-YZR and hygA-YZF/PoCyc8-DR respectively); lane 11 and lane 12 represent negative control (P. oxalicum 114-2). The PCR products were sequenced to verify the proper insertion of FLAG-HA tags. (C) Map of pMD18-T-NYFP which carries the N-terminal (1-155 aa) of the YFP. (D) Map of pUC19-NYFP which carries C-terminal (156-238 aa) of the YFP. (E) Results of diagnostic PCR of BiFC strains using primers NYZF/NYZR (lane 1, 3, 5, 7, 9, 11, 13) and CYZF/CYZR (lane 2, 4, 6, 8, 10, 12, 14). Lane 1 (5200 bp) and lane 2 (2740 bp) represent PoCyc8-YFP-PoCreA; lane 3 (5200 bp) and lane 4 (4789 bp) represent PoCyc8-YFP-PoSet2; lane 5 (5217 bp) and lane 6 (2740 bp) represent PoTup1-YFP-PoCreA; lane 7 (5200 bp) and lane 8 (1515 bp) represent PoCyc8-YFP-empty; lane 9 (5217 bp) and lane 10 (1515 bp) represent PoTup1-YFP-empty; lane 11 (2271 bp) and lane 12 (1515 bp) represent empty-YFP-empty; lane 13 and 14 were negative control amplified by template of the parent strain P. oxalicum 114-2. The PCR products were sequenced to verify the proper fusion of YFP fragments with the target proteins.

Additional file 5: Figure S3. The predicted protein-protein docking between PoCreA and PoTup1-Cyc8 complex. (A) The predicted PoTup1/ Cyc 8 docking model and predicted protein model of PoCreA, respectively. (B) The predicted PoCreA-Tup1/Cyc8 docking model. (C) The predicted PoCreA-Tup1/Cyc8 docking model was rotated $90^{\circ}$ clockwise vertically. (D) The predicted PoCreA-Tup1/Cyc8 docking model was rotated $90^{\circ}$ clockwise vertically twice.

Additional file 6: Figure S4. The original images of Western blot. (A) The anti-H3K4me1 antibody, anti-H3K4me2 antibody, and anti-H3K4me3 antibody were used to detect $\mathrm{H} 3 \mathrm{~K} 4$ methylation. (B) The anti-H3K36me1 antibody, anti-H3K36me2 antibody, and anti-H3K36me3 antibody were used to detect H3K36 methylation. (C) The anti-H3K79me1 antibody and anti-H3K79me2 antibody were used to detect H3K79 methylation. (D) Equal amounts of the total protein and the anti-histone $\mathrm{H} 3$ antibody were set as the loading control.

Additional file 7: Figure S5. Domain architecture analysis of Tup1 and Cyc8 in S. cerevisiae, T. reesei and P. oxalicum. (A) Domain architecture analysis of Cyc8 orthologs. (B) Domain architecture analysis of Tup1 orthologs. The SMART server (http://smart.embl-heidelberg.de/) was used for the domain architecture analysis of Tup $1 p$ and Cyc $8 p$ in S. cerevisiae, $P$. oxalicum, and T. reesei.

Additional file 8: Figure S6. The effects of CreA/Cre1 on the expression of set 1, set2, tup 1, and cyc 8 in T. reesei and M. grisea according to their transcriptome data. The transcription data were retrieved from Gene Expression Omnibus (https://www.ncbi.nlm.nih.gov/geo/). The datasets for T. reesei Trcre1 deletion strain are GSE57374. The datasets for M. grisea Mgcrel deletion strain are GSE153084.

Additional file 9: Table S2. Primers used in this study. 


\section{Acknowledgements}

We thank Senior Engineer Xuezhi Li and Dr. Jingyao Qu of the State Key Laboratory of Microbial Technology, Shandong University, as well as Xianggan Cui at Shanghai Bioprofile Technology Company Ltd. for helping BiFC observation and the MS data analysis.

\section{Authors' contributions}

$\mathrm{YH}$ performed the experiment and wrote the manuscript; $\mathrm{ML}$ contributed to data analysis; ZL contributed to manuscript correction; XS and YQu helped perform the analysis with constructive discussions. The corresponding author YQi contributed to the conception of the study. All authors read and approved the final manuscript.

\section{Funding}

This work was supported by the National Key Research and Development Project of China (Grant no. 2018YFA0900500), the National Natural Sciences Foundation of China (Grant No. 32070077, 31370086, 31971387), the Shandong Provincial Natural Science Foundation, China (Grant No. ZR2019MC007), the Major Basic Research Project of Natural Science Foundation of Shandong Province, China (Grant No. ZR2019ZD19) and the Central Government Guide Local Science and Technology Development Funds (Grant No. YDZX20203700002579).

\section{Availability of data and materials}

The information about the proteins of T. reesei on Table 1 are retrieved from the reference sequence (RefSeq) genome of T. reesei QM6a (Accession: PRJNA225530) (https://www.ncbi.nlm.nih.gov/bioproject/PRJNA225530). The information about the proteins of P. oxalicum on Table 2 and Table 3 are retrieved from the Whole Genome Shotgun project of P. oxalicum (Accession: AGIH00000000.1) (https://www.ncbi.nlm.nih.gov/nuccore/AGIH00000000.1). The information about the proteins of S. cerevisiae on Tables 1, 2, and 3 are retrieved from Saccharomyces Genome Database (www.yeastgenome.org). All other data that support the findings of this study can be found in Additional files $1,2,3,4,5,6,7,8$ and 9 .

\section{Declarations}

Ethics approval and consent to participate

Not applicable.

\section{Competing interests}

The authors declare that they have no competing interests.

\section{Author details}

${ }^{1}$ National Glycoengineering Research Center, State Key Laboratory of Microbial Technology, Shandong University, No. 72 Binhai Road, Qingdao 266237, China. ${ }^{2}$ Shandong Key Laboratory of Carbohydrate Chemistry and Glycobiology, Shandong University, No. 72 Binhai Road, Qingdao 266237, China. ${ }^{3}$ NMPA Key Laboratory for Quality Research and Evaluation of Carbohydrate-Based Medicine, Shandong University, No. 72 Binhai Road, Qingdao 266237, China.

Received: 2 June 2021 Accepted: 4 December 2021

Published online: 24 December 2021

\section{References}

1. Isikgor FH, Becer CR. Lignocellulosic biomass: a sustainable platform for the production of bio-based chemicals and polymers. Polym Chem-Uk. 2015;6:4497-559.

2. Berner RA. The long-term carbon cycle, fossil fuels and atmospheric composition. Nature. 2003;426:323-6.

3. Lynd LR, Liang X, Biddy MJ, Allee A, Cai H, Foust T, et al. Cellulosic ethanol: status and innovation. Curr Opin Biotech. 2017;45:202-11.

4. Mäkelä MR, Donofrio N, de Vries RP. Plant biomass degradation by fungi. Fungal Genet Biol. 2014;72:2-9.

5. Antonieto ACC, Castro LD, Silva-Rocha R, Persinoti GF, Silva RN. Defining the genome-wide role of CRE1 during carbon catabolite repression in Trichoderma reesei using RNA-Seq analysis. Fungal Genet Biol. 2014;73:93-103.
6. Adnan M, Zheng W, Islam W, Arif M, Abubakar YS, Wang Z, et al. Carbon catabolite repression in filamentous fungi. Int J Mol Sci. 2017;19:48.

7. Brown NA, Ries $L N A$, Goldman GH. How nutritional status signalling coordinates metabolism and lignocellulolytic enzyme secretion. Fungal Genet Biol. 2014;72:48-63.

8. Liu G, Zhang L, Qin Y, Zou G, Li Z, Yan X, et al. Long-term strain improvements accumulate mutations in regulatory elements responsible for hyper-production of cellulolytic enzymes. Sci Rep. 2013;3:1569.

9. Tamayo EN, Villanueva A, Hasper AA, de Graaff LH, Ramón D, Orejas M. CreA mediates repression of the regulatory gene $x \ln R$ which controls the production of xylanolytic enzymes in Aspergillus nidulans. Fungal Genet Biol. 2008;45:984-93.

10. Ries L, Belshaw NJ, IImen M, Penttila ME, Alapuranen M, Archer DB. The role of CRE1 in nucleosome positioning within the cbh1 promoter and coding regions of Trichoderma reesei. Appl Microbiol Biotechnol. 2014;98:749-62.

11. Strauss J, Mach RL, Zeilinger S, Hartler G, Stoffler G, Wolschek M, et al. Crel, the carbon catabolite repressor protein from Trichoderma-reesei. FEBS Lett. 1995;376:103-7.

12. Cziferszky A, Mach RL, Kubicek CP. Phosphorylation positively regulates DNA binding of the carbon catabolite repressor Cre1 of Hypocrea jecorina (Trichoderma reesei). J Biol Chem. 2002;277:14688-94.

13. de Assis LJ, Silva LP, Bayram O, Dowling P, Kniemeyer O, Krueger T, et al. Carbon catabolite repression in filamentous fungi is regulated by phosphorylation of the transcription factor CreA. MBio. 2021;12:e03146-20.

14. Lichius A, Seidl-Seiboth V, Seiboth B, Kubicek CP. Nucleo-cytoplasmic shuttling dynamics of the transcriptional regulators XYR1 and CRE1 under conditions of cellulase and xylanase gene expression in Trichoderma reesei. Mol Microbiol. 2014;94:1162-78.

15. Han L, Tan Y, Ma W, Niu K, Hou S, Guo W, et al. Precision engineering of the transcription factor Cre1 in Hypocrea jecorina (Trichoderma reesei) for efficient cellulase production in the presence of glucose. Front Bioeng Biotechnol. 2020;8:852.

16. Li Z, Yao G, Wu R, Gao L, Kan Q, Liu M, et al. Synergistic and dose-controlled regulation of cellulase gene expression in Penicillium oxalicum. PLoS Genet. 2015;11:e1005509.

17. Mach RL, Zeilinger S. Regulation of gene expression in industrial fungi: Trichoderma. Appl Microbiol Biotechnol. 2003;60:515-22.

18. Novy V, Schmid M, Eibinger M, Petrasek Z, Nidetzky B. The micromorphology of Trichoderma reesei analyzed in cultivations on lactose and solid lignocellulosic substrate, and its relationship with cellulase production. Biotechnol Biofuels. 2016:9:169.

19. Nakari-Setälä T, Paloheimo M, Kallio J, Vehmaanperä J, Penttilä M, Saloheimo M. Genetic modification of carbon catabolite repression in Trichoderma reesei for improved protein production. Appl Environ Microbiol. 2009;75:4853-60.

20. Peterson R, Nevalainen H. Trichoderma reesei RUT-C30-thirty years of strain improvement. Microbiology (Reading, England). 2012;158:58-68.

21. Cupertino FB, Virgilio S, Freitas FZ, Candido TD, Bertolini MC. Regulation of glycogen metabolism by the CRE-1, RCO-1 and RCM-1 proteins in Neurospora crassa. The role of $\mathrm{CRE}-1$ as the central transcriptional regulator. Fungal Genet Biol. 2015;77:82-94.

22. Díaz RD, Larrondo LF. A circadian clock in Neurospora crassa functions during plant cell wall deconstruction. Fungal Biol. 2020;124:501-8.

23. Luo Z, Qin Y, Pei Y, Keyhani NO. Ablation of the creA regulator results in amino acid toxicity, temperature sensitivity, pleiotropic effects on cellular development and loss of virulence in the filamentous fungus Beauveria bassiana. Environ Microbiol. 2014;16:1122-36.

24. Zhang X, Qu Y, Qin Y. Expression and chromatin structures of cellulolytic enzyme gene regulated by heterochromatin protein 1. Biotechnol Biofuels. 2016;9:206.

25. Klein CJ, Olsson L, Rønnow B, Mikkelsen JD, Nielsen J. Alleviation of glucose repression of maltose metabolism by MIG1 disruption in Saccharomyces cerevisiae. Appl Environ Microbiol. 1996;62:4441-9.

26. Treitel MA, Carlson M. Repression by SSN6-TUP1 is directed by MIG1, a repressor/activator protein. Proc Natl Acad Sci USA. 1995;92:3132-6.

27. García I, Mathieu M, Nikolaev I, Felenbok B, Scazzocchio C. Roles of the Aspergillus nidulans homologues of Tup1 and Ssn6 in chromatin structure and cell viability. FEMS Microbiol Lett. 2008;289:146-54. 
28. Hicks J, Lockington RA, Strauss J, Dieringer D, Kubicek CP, Kelly J, et al. RcoA has pleiotropic effects on Aspergillus nidulans cellular development. Mol Microbiol. 2001;39:1482-93.

29. Olmedo M, Navarro-Sampedro L, Ruger-Herreros C, Kim SR, Jeong BK, Lee $\mathrm{BU}$, et al. A role in the regulation of transcription by light for RCO-1 and RCM-1, the Neurospora homologs of the yeast Tup1-Ssn6 repressor Fungal Genet Biol. 2010;47:939-52.

30. Wang L, Zhang W, Cao Y, Zheng F, Zhao G, Lv X, et al. Interdependent recruitment of CYC8/TUP1 and the transcriptional activator XYR1 at target promoters is required for induced cellulase gene expression in Trichoderma reesei. PLoS Genet. 2021;17:e1009351.

31. Zhong L, Qian Y, Dai M, Zhong Y. Improvement of uracil auxotrophic transformation system in Trichoderma reesei QM9414 and overexpression of $\beta$-glucosidase. CIESC J. 2016; 67:2510-8.

32. Ishihama Y, Oda Y, Tabata T, Sato T, Nagasu T, Rappsilber J, et al. Exponentially modified protein abundance index (emPAl) for estimation of absolute protein amount in proteomics by the number of sequenced peptides per protein. Mol Cell Proteomics MCP. 2005;4:1265-72.

33. Tanaka M, Ichinose S, Shintani T, Gomi K. Nuclear export-dependent degradation of the carbon catabolite repressor CreA is regulated by a region located near the C-terminus in Aspergillus oryzae. Mol Microbiol. 2018;110:176-90.

34. Zhang X, Li M, Zhu Y, Yang L, Li Y, Qu J, et al. Penicillium oxalicum putative methyltransferase Mtr23B has similarities and differences with LaeA in regulating conidium development and glycoside hydrolase gene expression. Fungal Genet Biol. 2020;143:103445.

35. Hu CD, Chinenov Y, Kerppola TK. Visualization of interactions among bZIP and Rel family proteins in living cells using bimolecular fluorescence complementation. Mol Cell. 2002;9:789-98.

36. Waterhouse A, Bertoni M, Bienert S, Studer G, Tauriello G, Gumienny $\mathrm{R}$, et al. SWISS-MODEL: homology modelling of protein structures and complexes. Nucleic Acids Res. 2018;46:W296-w303.

37. Yan Y, Zhang D, Zhou P, Li B, Huang SY. HDOCK: a web server for protein-protein and protein-DNA/RNA docking based on a hybrid strategy. Nucleic Acids Res. 2017;45:W365-w373.

38. Carrozza MJ, Li B, Florens L, Suganuma T, Swanson SK, Lee KK, et al. Histone $\mathrm{H} 3$ methylation by Set2 directs deacetylation of coding regions by Rpd3S to suppress spurious intragenic transcription. Cell. 2005;123:581-92.

39. Li Y, Hu Y, Zhao K, Pan Y, Qu Y, Zhao J, et al. The indispensable role of histone methyltransferase PoDot1 in extracellular glycoside hydrolase biosynthesis of Penicillium oxalicum. Front Microbiol. 2019;10:2566.

40. Li Y, Hu Y, Zhu Z, Zhao K, Liu G, Wang L, et al. Normal transcription of cellulolytic enzyme genes relies on the balance between the methylation of H3K36 and H3K4 in Penicillium oxalicum. Biotechnol Biofuels. 2019:12:198.

41. Seiboth B, Karimi RA, Phatale PA, Linke R, Kubicek CP. The putative protein methyltransferase LAE1 controls cellulase gene expression in Trichoderma reesei. Mol Microbiol. 2012;84:1150-64.

42. Vu BV, Pham KT, Nakayashiki H. Substrate-induced transcriptional activation of the MoCel7C cellulase gene is associated with methylation of histone $\mathrm{H} 3$ at lysine 4 in the rice blast fungus Magnaporthe oryzae. Appl Environ Microbiol. 2013;79:6823-32.

43. Lai Y, Cao X, Chen J, Wang L, Wei G, Wang S. Coordinated regulation of infection-related morphogenesis by the KMT2-Cre1-Hyd4 regulatory pathway to facilitate fungal infection. Sci Adv. 2020;6:eaaz1659.

44. Kayikci O, Nielsen J. Glucose repression in Saccharomyces cerevisiae. FEMS Yeast Res. 2015;15:fov068.

45. Znameroski EA, Coradetti ST, Roche CM, Tsai JC, lavarone AT, Cate JHD, et al. Induction of lignocellulose-degrading enzymes in Neurospora crassa by cellodextrins. Proc Natl Acad Sci USA. 2012;109:6012-7.

46. Li J, Moazed D, Gygi SP. Association of the histone methyltransferase Set2 with RNA polymerase II plays a role in transcription elongation. J Biol Chem. 2002;277:49383-8.

47. Roguev A, Schaft D, Shevchenko A, Pijnappel WWMP, Wilm M, Aasland R, et al. The Saccharomyces cerevisiae Set1 complex includes an Ash2 homologue and methylates histone 3 lysine 4. Embo J. 2001:20:7137-48

48. Tripic T, Edmondson DG, Davie JK, Strahl BD, Dent SY. The Set2 methyltransferase associates with Ssn6 yet Tup1-Ssn6 repression is independent of histone methylation. Biochem Biophys Res Commun. 2006;339:905-14.

49. Watson JD, Baker TA, Bell SP, Gann A. Mastering biology with pearson Etext-Valuepack access card_for molecular biology of the gene. 2013.

50. Silva-Rocha R, Castro LD, Antoniero ACC, Guazzaroni ME, Persinoti GF, Silva RN. Deciphering the cis-regulatory elements for XYR1 and CRE1 regulators in Trichoderma reesei. PLoS ONE. 2014;9:e99366.

51. Castro Ldos S, Antoniêto AC, Pedersoli WR, Silva-Rocha R, Persinoti GF, Silva RN. Expression pattern of cellulolytic and xylanolytic genes regulated by transcriptional factors XYR1 and CRE1 are affected by carbon source in Trichoderma reesei. Gene Expr Patterns GEP. 2014;14:88-95.

52. Kadonaga JT. The transformation of the DNA template in RNA polymerase II transcription: a historical perspective. Nat Struct Mol Biol. 2019;26:766-70.

53. Li B, Carey M, Workman JL. The role of chromatin during transcription. Cell. 2007;128:707-19.

54. Alam MA, Kelly JM. Proteins interacting with CreA and CreB in the carbon catabolite repression network in Aspergillus nidulans. Curr Genet. 2017;63:669-83.

55. Gounalaki N, Tzamarias D, Vlassi M. Identification of residues in the TPR domain of Ssn6 responsible for interaction with the Tup1 protein. FEBS Lett. 2000;473:37-41.

56. Varanasi US, Klis M, Mikesell PB, Trumbly RJ. The Cyc8 (Ssn6)-Tup1 corepressor complex is composed of one Cyc8 and four Tup1 subunits. Mol Cell Biol. 1996;16:6707-14.

57. Smith RL, Johnson AD. Turning genes off by Ssn6-Tup1: a conserved system of transcriptional repression in eukaryotes. Trends Biochem Sci. 2000;25:325-30.

58. Zeilinger S, Schmoll M, Pail M, Mach RL, Kubicek CP. Nucleosome transactions on the Hypocrea jecorina (Trichoderma reesei) cellulase promoter cbh2 associated with cellulase induction. Mol Genet Genomics. 2003:270:46-55.

59. Hong Y, Cai R, Guo J, Zhong Z, Bao J, Wang Z, et al. Carbon catabolite repressor MoCreA is required for the asexual development and pathogenicity of the rice blast fungus. Fungal Genet Biol. 2021;146:103496.

60. Tripic T, Edmondson DG, Davie JK, Strahl BD, Dent SYR. The Set2 methyltransferase associates with Ssn6 yet Tup1-Ssn6 repression is independent of histone methylation. Biochem Bioph Res Commun. 2006:339:905-14.

61. Bannister AJ, Kouzarides T. Regulation of chromatin by histone modifications. Cell Res. 2011;21:381-95.

62. Kim JH, Lee BB, Oh YM, Zhu C, Steinmetz LM, Lee YY, et al. Modulation of mRNA and IncRNA expression dynamics by the Set2-Rpd3S pathway. Nat Commun. 2016;7:13534.

63. Lee BB, Kim JH, Kim T. Fine-tuning of gene expression dynamics by the Set2-Rpd3S pathway. BMB Rep. 2017;50:162-3.

64. Green SR, Johnson AD. Promoter-dependent roles for the Srb10 cyclin-dependent kinase and the Hda1 deacetylase in Tup1-mediated repression in Saccharomyces cerevisiae. Mol Biol Cell. 2004;15:4191-202.

65. Gromoller A, Lehming N. Srb7p is a physical and physiological target of Tup1p. Embo J. 2000;19:6845-52.

66. Papamichos-Chronakis M, Conlan RS, Gounalaki N, Copf T, Tzamarias D. Hrs1/Med3 is a Cyc8-Tup1 corepressor target in the RNA polymerase II holoenzyme. J Biol Chem. 2000;275:8397-403.

67. Cramer P. Multisubunit RNA polymerases. Curr Opin Struct Biol. 2002;12:89-97.

68. Watson AD, Edmondson DG, Bone JR, Mukai Y, Yu Y, Du W, et al. Ssn6Tup1 interacts with class I histone deacetylases required for repression. Genes Dev. 2000;14:2737-44.

69. Chia M, Li C, Marques S, Pelechano V, Luscombe NM, van Werven FJ. High-resolution analysis of cell-state transitions in yeast suggests widespread transcriptional tuning by alternative starts. Genome Biol. 2021;22:34

70. Vogel HJ. A convenient growth medium for Neurospora crassa. Microb Genet Bull. 1956;13:42-3.

71. Qin Y, Bao L, Gao M, Chen M, Lei Y, Liu G, et al. Penicillium decumbens BrlA extensively regulates secondary metabolism and functionally associates with the expression of cellulase genes. Appl Microbiol Biotechnol. 2013;97:10453-67. 
72. Nakayashiki H, Hanada S, Nguyen BQ, Kadotani N, Tosa Y, Mayama S RNA silencing as a tool for exploring gene function in ascomycete fungi. Fungal Genet Biol. 2005;42:275-83.

73. Sun X, Liu Z, Qu Y, Li X. The effects of wheat bran composition on the production of biomass-hydrolyzing enzymes by Penicillium decumbens. Appl Biochem Biotechnol. 2008;146:119-28.

74. Khramtsov P, Kalashnikova T, Bochkova M, Kropaneva M, Timganova V, Zamorina S, et al. Measuring the concentration of protein nanoparticles synthesized by desolvation method: comparison of Bradford assay, BCA assay, hydrolysis/UV spectroscopy and gravimetric analysis. Int J Pharm. 2021;599:120422

75. Chevallet M, Luche S, Rabilloud T. Silver staining of proteins in polyacrylamide gels. Nat Protoc. 2006;1:1852-8.

\section{Publisher's Note}

Springer Nature remains neutral with regard to jurisdictional claims in published maps and institutional affiliations.

- fast, convenient online submission

- thorough peer review by experienced researchers in your field

- rapid publication on acceptance

- support for research data, including large and complex data types

- gold Open Access which fosters wider collaboration and increased citations

- maximum visibility for your research: over $100 \mathrm{M}$ website views per year

At $\mathrm{BMC}$, research is always in progress.

Learn more biomedcentral.com/submissions 